\title{
Large-Scale Circulation and Production of Stratification: Effects of Wind, Geometry, and Diffusion
}

\author{
GEOFFREY K. VALLIS \\ GFDL and Princeton University, Princeton, New Jersey
}

(Manuscript received 20 January 1998, in final form 20 May 1999)

\section{ABSTRACT}

\begin{abstract}
The combined effects of wind, geometry, and diffusion on the stratification and circulation of the ocean are explored by numerical and analytical methods. In particular, the production of deep stratification in a simply configured numerical model with small diffusivity is explored.

In the ventilated thermocline of the subtropical gyre, the meridional temperature gradient is mapped continously to a corresponding vertical profile, essentially independently of (sufficiently small) diffusivity. Below this, as the vertical diffusivity tends to zero, the mapping becomes discontinuous and is concentrated in thin diffusive layers or internal thermoclines. It is shown that the way in which the thickness of the main internal thermocline (i.e., the diffusive lower part of the main thermocline), and the meridional overturning circulation, scales with diffusivity differs according to the presence or absence of a wind stress. For realistic parameter values, the ocean is in a scaling regime in which wind effects are important factors in the scaling of the thermohaline circulation, even for the single hemisphere, flat-bottomed case.

It is shown that deep stratification may readily be produced by the combined effects of surface thermodynamic forcing and geometry. The form of the stratification, but not its existence, depends on the diffusivity. Such deep stratification is efficiently produced, even in single-basin, single-hemisphere simulations, in the presence of a partially topographically blocked channel at high latitudes, provided there is also a surface meridional temperature gradient across the channel. For sufficiently simple geometry and topography, the abyssal stratification is a maximum at the height of the topography. In the limit of small diffusivity, the stratification becomes concentrated in a thin diffusive layer, or front, whose thickness appears to scale as the one-third power of the diffusivity. Above and below this diffusive abyssal thermocline are thick, largely adiabatic and homogeneous water masses. In two hemisphere integrations, the water above the abyssal thermocline may be either "intermediate" water from the same hemisphere as the channel, or "deep" water from the opposing hemisphere, depending on whether the densest water from the opposing hemisphere is denser than the surface water at the equatorward edge of the channel. The zonal velocity in the channel is in thermal wind balance, thus determined more by the meridional temperature gradient across the channel than by the wind forcing. If the periodic channel extends equatorward past the latitude of zero wind-stress curl, the poleward extent of the ventilated thermocline, and the surface source of the mode water, both then lie at the equatorial boundary of the periodic circumpolar channel, rather than where the wind stress curl changes sign.
\end{abstract}

\section{Introduction}

This paper seeks to make progress in the task of understanding the factors determining the stratification of the ocean, by way of numerical experimentation with an ocean model and, to a lesser degree, analytic methods. Our approach is motivated by a number of previous studies using the planetary geostrophic (PG) equations, by various primitive equation (PE) experiments using ocean general circulation models, and by numerous observations of the density structure of the ocean. Building on prior work by Colin de Verdiere $(1988,1989)$ and Salmon (1986, 1990), Samelson and Vallis (1997a,b,

Corresponding author address: Dr. Geoffrey K. Vallis, AOS Program, Princeton University, Princeton, NJ 08544.

E-mail: gkv@princeton.edu henceforth SV) explored the circulation of a planetary geostrophic ocean model in a single basin, concentrating on the density structure of the upper ocean. Perhaps their most significant result was that there are two distinct dynamical regimes in the thermocline. On isopycnals that outcrop in the subtropical gyre the balance in the thermodynamic equation is essentially adiabatic: a ventilated thermocline on which potential vorticity is conserved on parcels determines the stratification (Luyten et al. 1983). [The development of an adiabatic theory of the thermocline began with Welander $(1959,1971 \mathrm{a})$ and Veronis $(1969,1973)$. Huang $(1988$, and in subsequent papers) developed a continuous extension to the theory.] Immediately below the ventilated thermocline lies an "internal boundary layer," as discussed by Stommel and Webster (1962), Salmon (1990), and Young and Ierley (1986), and this forms an "internal thermocline," 
or front. Indeed the so-called thermocline equations are essentially the planetary geostrophic equations, plus a diffusive term. The existence of an internal thermocline is demanded because the water at the base of the ventilated thermocline has different thermodynamic properties than the abyssal water, and therefore there must be a transition region, or boundary layer, which in general must depend on diffusivity. For realistically small values of the diffusivity the transition region is relatively thin (e.g., of order a few hundreds of meters) compared to the depth of the ocean, and can be dynamically and physically distinguished from the ventilated thermocline above it. The ventilated thermocline and the internal thermocline together form the "main" thermocline. Based on studies with a PG model, SV suggested that the thickness of the internal thermocline be proportional to the half-power of the diffusivity. The half-power dependence of the thickness of the thermocline is different from that found in some other simulations (e.g., Bryan 1987; Zhang et al. 1992) and that of classical scaling (see section 3). Marotzke (1997) and Park and Bryan (2000) have both, in different contexts, recently revisited the issue of effects of diffusivity (although without discussing wind effects). All these studies were performed by different models or with different forcing and parameters, and because the differences in scaling regimes is quantitatively small, it remains unclear as to whether and how the wind really does affect the scaling. Note that the effect of the wind is (potentially) not only to transport heat in wind-driven gyres, but also to affect the scaling of the meridional overturning circulation and its associated heat transport.

In nearly all of the idealized simulations mentioned above, the deep stratification of the model ocean is typically much less than that observed. With small diffusivity, there is no process to efficiently heat the abyss below the internal thermocline, and the consequence is that the abyss fills with the densest available water, typically that from high latitudes. Similar effects have been noted in primitive equation models (see Cummins 1991; Cummins et al. 1990). However, the use of realistic geometry and topography does seem to affect the thermohaline circulation and stratification of ocean models. In particular Gill and Bryan (1971) and Cox (1989) noted the large effects of topography and the Drake Passage on the global thermohaline circulation, although these simulations had relatively large diffusivity that obscured the adiabatic production of deep stratification. England (1993) and Toggweiler and Samuels (1995) examined related issues in more geographically complete simulations, also with relatively high vertical diffusivity. Another possible influence on the deep stratification is locally intense mixing (Samelson 1998); mixing at or near boundaries may be orders of magnitude more efficient than that in the open ocean, and therefore might cause deep stratification. However, because of the uncertain nature of diapycnal mixing in the ocean, it seems important to determine whether deep stratification can be produced without invoking diffusive effects.

In this paper we explore some of these issues further with a standard PE model of the ocean circulation, namely the Modular Ocean Model (MOM: Pacanowski 1996). Specific questions we wish to address are

1) How is the scaling of the thermocline thickness and meridional overturning circulation affected by wind? Does a primitive equation numerical model in fact reproduce the scalings suggested by theory for the thermocline thickness and the meridional overturnings?

2) Given a uniformly small diffusivity, can we obtain deep stratification? What is the simplest model configuration that will produce deep stratification and how is the stratification affected by changes in the geometry, including the width of the channel and the presence of two hemispheres?

Although the particular dependence of the thermocline thickness and the meridional overturning circulation on the precise power of the diffusivity may seem an arcane point, it is a reflection of our understanding of thermocline structure and of the general circulation. It has been the subject of a number of papers, although none have explicitly explored the scaling both with and without wind. A secondary and more general goal of this paper is that of making a connection between analytic theories of the ocean circulation and more complete general circulation models that do not lend themselves to intuitive understanding, yet which do allow direct comparison with observation.

The structure of the paper is as follows. In the next section we briefly describe the model formulation. This is followed in section 3 by a description of the experiments on thermocline structure and scaling. Section 4 describes the experiments with varying geometry, and section 5 concludes.

\section{Model formulation}

The numerical experiments described in subsequent sections are performed with MOM (Pacanowski 1996). The reasons for using a primitive equation model like MOM rather than a planetary geostrophic (PG) model are twofold:

1) By using very standard equations and parameterizations, different in detail from those used in previous PG integrations, we demonstrate the reproducibility and assure robustness of results obtained.

2) A PE model affords more flexibility in configuration than a PG model, particularly in terms of adding topography. A PG model with topography requires the solution of an elliptic equation at every time step, losing much of its computational advantage over a PE model.

The disadvantages are that a PE model is still more 
computationally expensive and that very low values of diffusivity are not as easily available without the model becoming unsteady: although the growth rate of baroclinic instability of the inviscid PG equations grows linearly as the wave number increases (Colin de Verdiere 1986), at deformation scales the growth rate is small compared to that of a PE or quasigeostrophic model (Smith and Vallis 1999) and is easily controlled with a modest scale-dependent friction or diffusivity.

The winds used are zonally averaged, Hellerman and Rosenstein (1983). Restoring boundary conditions (to observed zonally averaged Levitus values) are used on both temperature and salinity, with a relaxation timescale of about 50 days on the uppermost model layer. This is shorter than most dynamical timescales in the model, resulting in a surface temperature distribution largely governed, except in the western boundary current or when very large values of diffusivity are used, by the imposed surface temperature distribution. Horizontal resolution is typically $2^{\circ}$ for the single-hemisphere experiments, $2.4^{\circ}$ in experiments involving two hemispheres. In the vertical about 26 unequally spaced levels are typically used, with a resolution of about 35 $\mathrm{m}$ near the surface, although 45 levels are used in the experiments with topography that explore diffusivity scaling. In the experiments involving main thermocline scaling and no topography, the vertical resolution remains high through the thermocline, but the grid-size increases to about $1000 \mathrm{~m}$ in the abyss. In the experiments with topography the abyssal grid size remains less than $250 \mathrm{~m}$ throughout the column. The vertical diffusivity is varied, but will be as small as $10^{-5} \mathrm{~m}^{2} \mathrm{~s}^{-1}$ $\left(0.1 \mathrm{~cm}^{2} \mathrm{~s}^{-1}\right)$. In all experiments it is spatially uniform. The horizontal diffusivity is typically of order $10^{2} \mathrm{~m}^{2}$ $\mathrm{s}^{-1}\left(10^{6} \mathrm{~cm}^{2} \mathrm{~s}^{-1}\right)$. Centered differencing is used throughout to minimize numerical diffusion. Strictly, the time needed for the model to be assured of reaching a complete equilibrium will be the longer of the diffusive time and advective time, and of these the former is usually larger. The diffusive timescale is given by $T_{\text {diff }} \sim D^{2} / k_{\nu}$, which for $k_{\nu}=0.1 \mathrm{~cm}^{2} \mathrm{~s}^{-1}$ and $D=5 \mathrm{~km}$, is about $80000 \mathrm{yr}$, beyond our computational resources. However, advective processes can accelerate equilibration; the advective timescale is $L / V$ and with $L=10^{7} \mathrm{~m}$ and $V=0.02 \mathrm{~cm} \mathrm{~s}^{-1}$ a timescale of about $1500 \mathrm{yr}$ is implied. In practice, the deep ocean will only require a diffusive time to reach equilibrium if its initial temperature is colder than the water that will eventually fill it, namely polar water. On the other hand, the thermocline, if its structure is least in part determined by diffusive processes, will require a diffusive time to equilibrate; this, supposing the thermocline to be $1 \mathrm{~km}$ thick, is approximately $3000 \mathrm{yr}$ for $k_{\nu}=0.1 \mathrm{~cm}^{2} \mathrm{~s}^{-1}$.

Bearing in mind the above considerations the following strategy was followed to reach equilibrium in the most efficient manner: any given geometric configuration of the model was initially integrated for about 4000 years with a high diffusivity (say $1 \mathrm{~cm}^{2} \mathrm{~s}^{-1}$ ). The dif- fusivity is then reduced to the value required and the model further integrated for about 2000 years. Further, in cases where a sequence of experiments was performed, the end state of one experiment formed the initial state of another; in such cases the need for the initial spinup period is somewhat reduced. In all cases reported, the model properties are changing only extremely slowly at the end of such a period, and for all practical purposes can be regarded as being in equilibrium. Some of the integrations, especially those with small horizontal and large vertical diffusivity, show a small jitter in a time series of a local quantity (like temperature at a grid point). However, in no cases do the fields shown in the figures differ qualitatively from those at another time.

\section{Thermocline structure}

\section{a. Qualitative preliminaries}

The model is first configured in a flat-bottomed single-basin sector domain, from $6^{\circ} \mathrm{N}$ to $70^{\circ} \mathrm{N}$ and with a longitudinal extent of $64^{\circ}$, so that its size and latitude roughly correspond to that of the North Atlantic, and the model integrated to a steady state. The model abyss has a very small stratification (Fig. 1), with a temperature equal to the surface temperature at the poleward extreme of the domain. In contrast, the upper ocean has a more complex pattern of stratification. In particular, toward the poleward end of the subtropical gyre the stratification (as measured, say, by $\partial T / \partial z$ or $N^{2}$ ) of the thermocline has two distinct maxima that merge together at lower latitudes. As mentioned, salinity is present with the same diffusivity and restoring boundary conditions as temperature, but its effects are found to be relatively small on the dynamical features that concern us in this paper. (For example, the main thermocline corresponds to a pycnocline; the temperature profile is not everywhere quite monotonic in the vertical, but the density profile is.)

The upper near-surface maxima of stratification is coincident with isotherms that outcrop in the subtropical gyre and is the manifestation of a ventilated thermocline (Luyten et al. 1983). The ventilated thermocline is (inter alia) a continuous mapping of the surface meridional temperature gradient in the subtropical gyre to a vertical temperature gradient. The dynamics in this regime are quasi adiabatic and quasi inviscid and therefore parcels that are subducted in the subtropical gyre conserve their potential vorticity. The upper maximum in the stratification shown in Fig. 1 is associated with a ventilated thermocline (cf. Cox and Bryan 1984). The forcing is time independent in these integrations, so the upper maxima in stratification is not a seasonal thermocline.

The bottom part of the ventilated thermocline comprises a thick thermostad. The mechanisms of its formation were discussed by SV, who associated this with convectively formed subtropical "mode water" (Mc- 


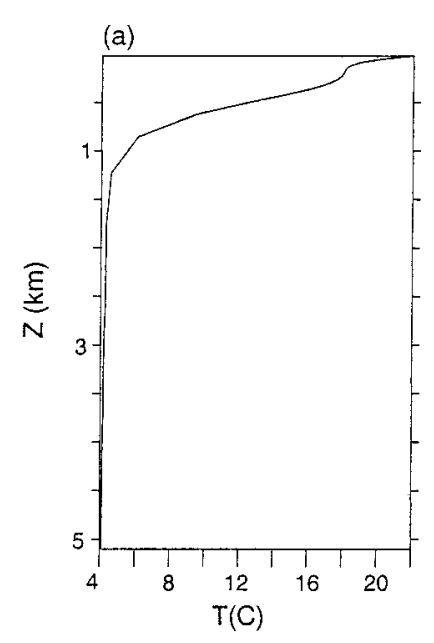

(b)
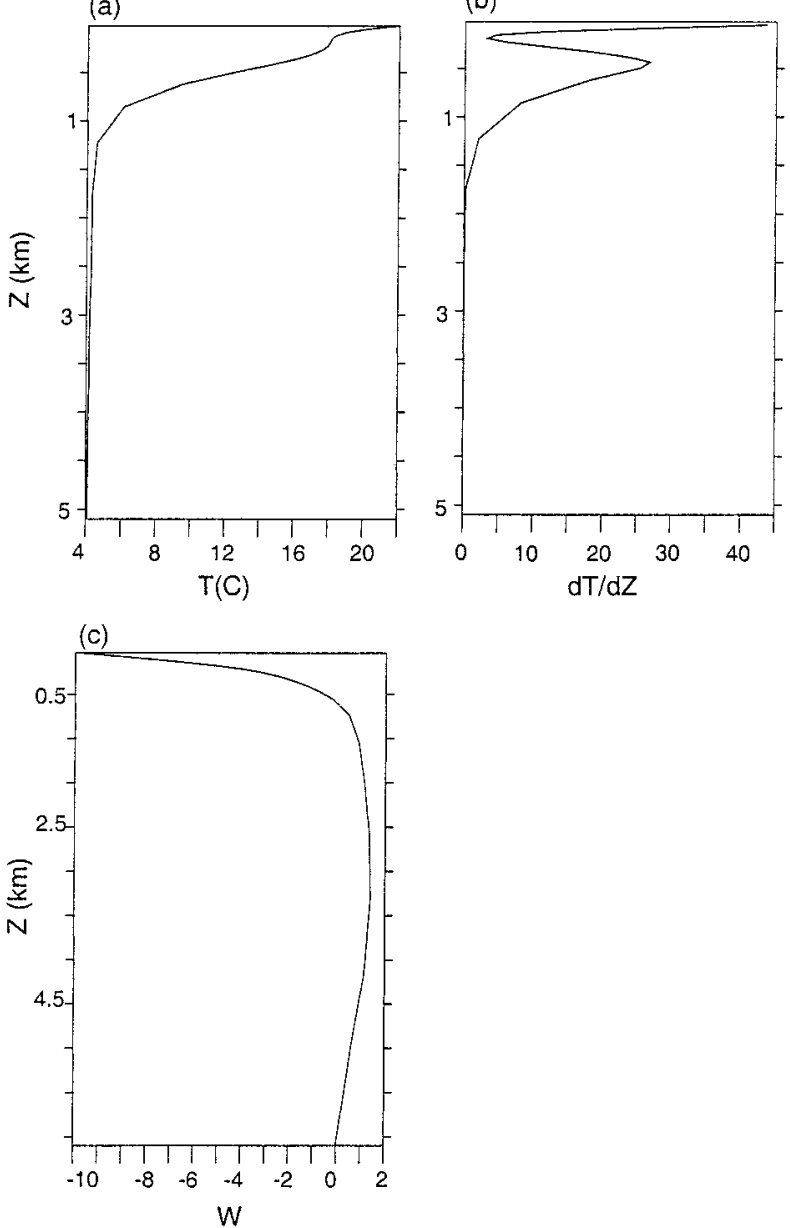

FIG. 1. Profiles of $T$ (a), $\partial T / \partial z$ (b), and vertical velocity (c), in the middle of the subtropical gyre, in a closed single-basin, flat-bottomed integration.

Cartney 1982; McCartney and Talley 1982). In the simulation here its temperature is indeed close to $18^{\circ}$, even though seasonality is not present in the simulations, so quantitative correspondence may be fortuitous. Although mode water forms a region of small stratification, its dynamics are largely adiabatic and it properly should be considered as part of the ventilated thermocline (even thought the moniker "cline" may be a little inappropriate when talking about a thermostad). The water at the base of the ventilated thermocline is not, in general, at the same temperature as the water in the abyss. Thus, in general, there must be a transition or matching region; this is the internal thermocline, as discussed in the next subsection.

\section{b. Wind and diffusivity scaling}

Following Welander (1971b) (see also SV), a scaling for the depth of the wind-driven influence can be derived by using the thermal wind, mass continuity, and ther-

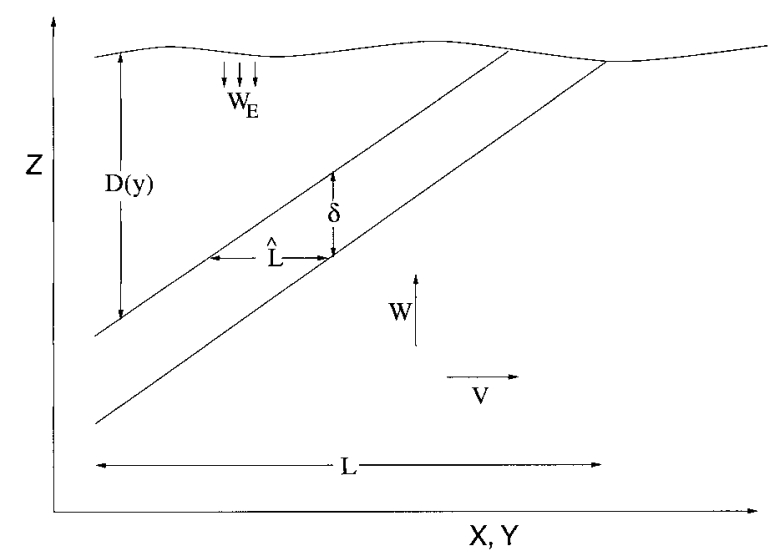

FIG. 2. Schema of the internal thermocline, indicating the basis for the scaling theory.
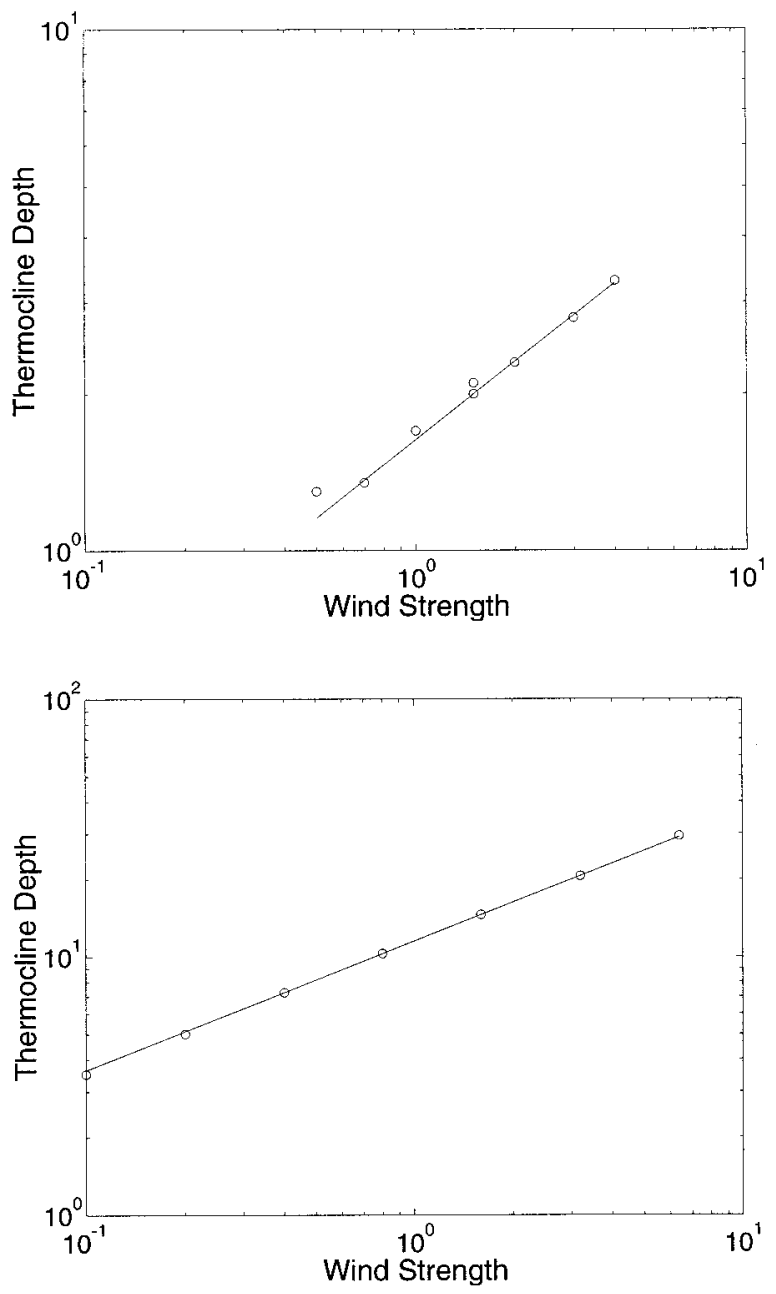

FIG. 3. (a) Thermocline depth (arbitrary units) as a function of wind strength, from MOM. The wind profile is the same in all cases; the numbers in the abscissae refer to a multiplying factor. The value 1 corresponds to the zonally averaged Hellerman and Rosenstein winds. The solid line is (wind strength) ${ }^{1 / 2}$. Single-basin, single hemisphere. (b) As for (a) but as obtained from numerical solutions of the similarity equation (3.16). 

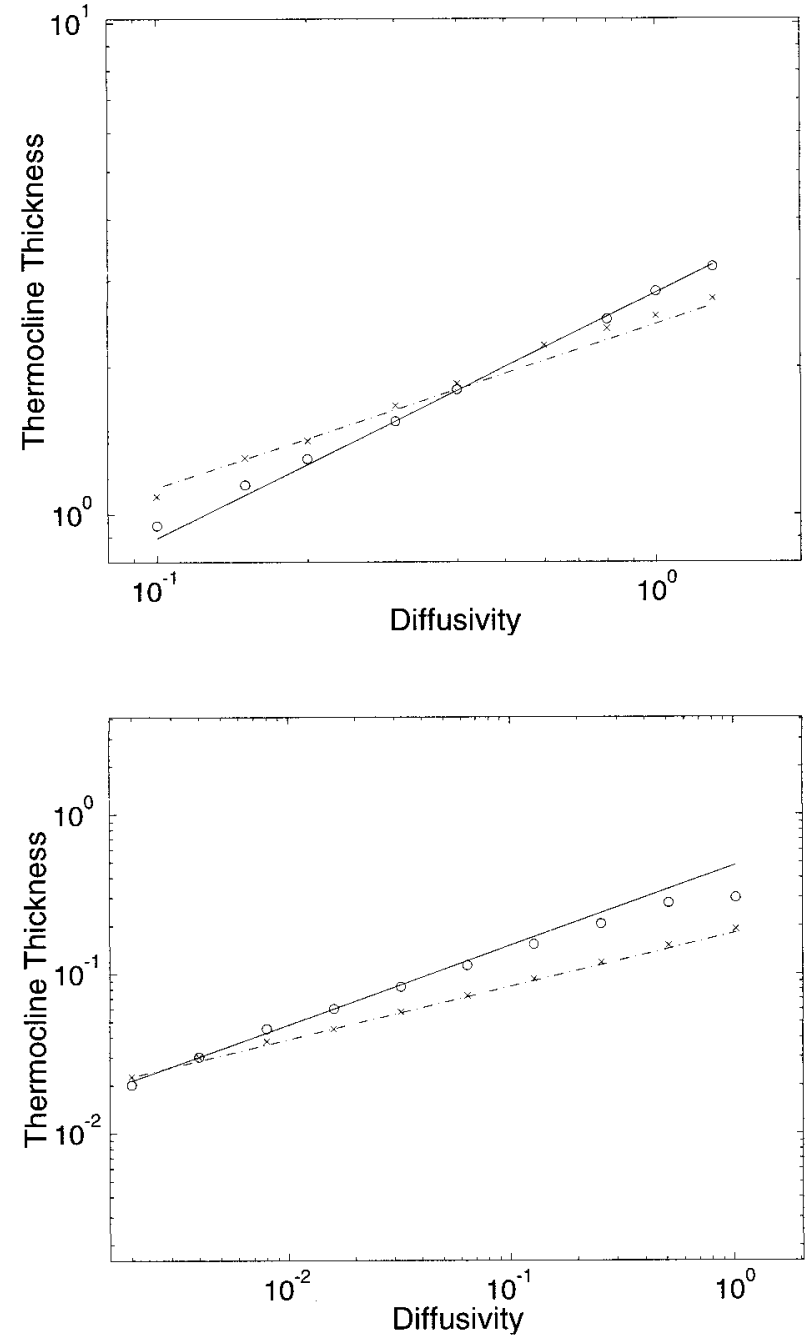

FIG. 4. (a) Internal thermocline thickness (arbitrary units) as a function of vertical diffusivity, from a single-basin integration in MOM. The circles refer to integrations with a standard wind forcing, and the crosses refer to integrations with zero wind. The solid and dashed lines are, respectively, proportional to $k_{\nu}^{1 / 2}$ and $k_{\nu}^{1 / 3}$. (b) As for (a) but as obtained by numerical solutions of the similarity solution (3.16).

modynamic equations. Possible scaling relationships these equations imply are, respectively,

$$
\begin{aligned}
\frac{f V}{D} & \sim g \alpha \frac{\Delta T}{L} \\
\frac{V}{L} & \sim \frac{W_{E}}{D} \\
\frac{V \Delta T}{L} & \sim \frac{W_{E} \Delta T}{D} .
\end{aligned}
$$

In these equations $W_{E}$ is the imposed Ekman pumping velocity, $\Delta T$ is the imposed meridional temperature difference across the thermocline, $V$ is a horizontal velocity scale, and $D$ and $L$ are vertical and horizontal length
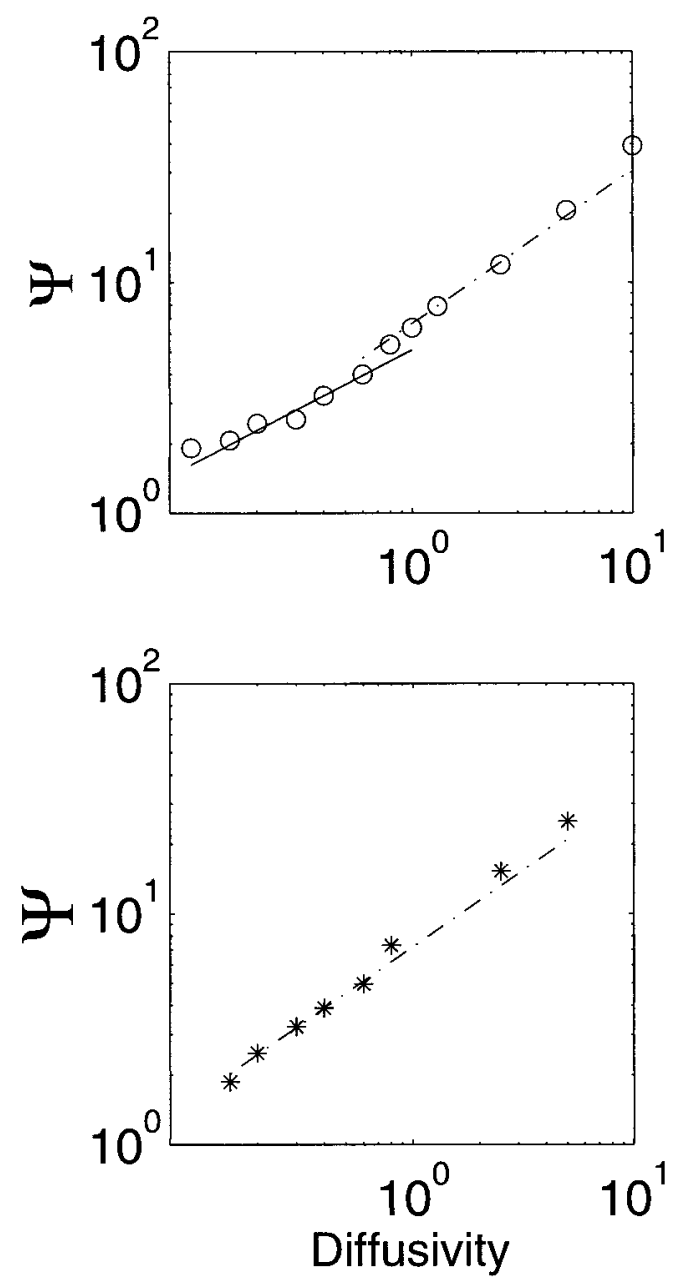

FIG. 5. Upper panel: maximum meridional overturning circulation in center of domain from a single basin integration in MOM with wind forcing. The solid and dashed lines are, respectively, proportional to $k_{v}^{1 / 2}$ and $k_{v}^{2 / 3}$. Lower panel: results from a similar set of integrations but with no wind forcing.

scales, respectively (Fig. 2). The thermodynamic equation is assumed to be adiabatic, and the vertical velocity in the thermodynamic and mass continuity equations is assumed to be the Ekman pumping velocity $W_{E}$. These equations easily yield

$$
D \sim\left\{\frac{W_{E} f L^{2}}{g \alpha \Delta T}\right\}^{1 / 2} \propto W_{E}^{1 / 2}
$$

This scaling can be equivalently regarded as giving either the depth of internal thermocline or the thickness of the ventilated thermocline. To test this, a sequence of experiments was performed with varying winds and a constant vertical diffusivity of $k_{\nu}=0.3 \mathrm{~cm}^{2} \mathrm{~s}^{-1}$. (Such a value is small enough to give an unambiguous definition of the thermocline depth, yet large enough to ensure absolutely steady results and reasonable equilibration times. Some tests were made with different values of diffusivity, and the dependence of depth on dif- 


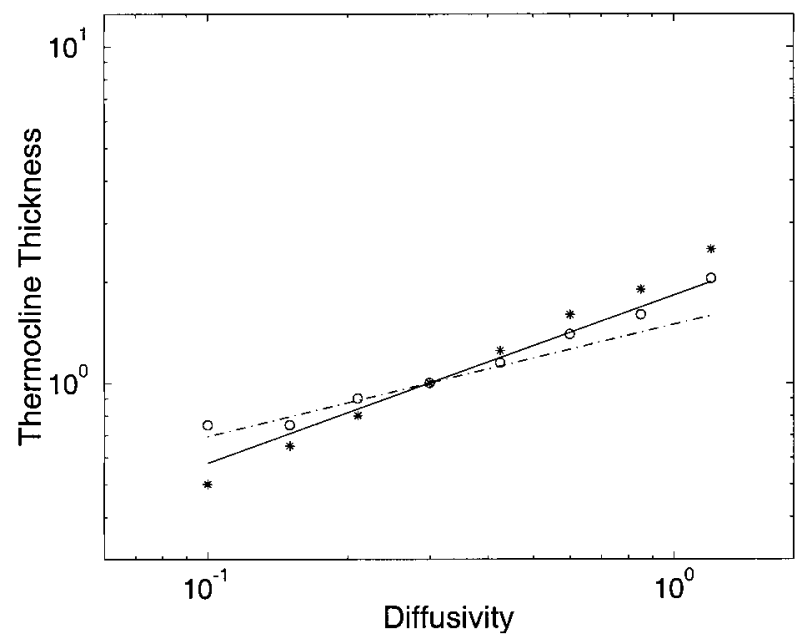

FIG. 6. Internal thermocline thickness (arbitrary units) as a function of vertical diffusivity $\left(\mathrm{cm}^{2} \mathrm{~s}^{-1}\right)$, from a two-hemisphere, single-basin integration in MOM. The circles are Northern Hemisphere results, the stars from the Southern Hemisphere. The solid line is proportional to $k_{\nu}^{1 / 2}$.

fusivity was found to be small.) The spatial variation of the winds is identical in all cases (it is in fact taken from observation), multiplied by a constant factor $(1 / 2$, $3 / 4,1,2,4)$. The depth of the internal maximum in $\partial T / \partial Z$ serves as a measure of the depth of the thermocline.
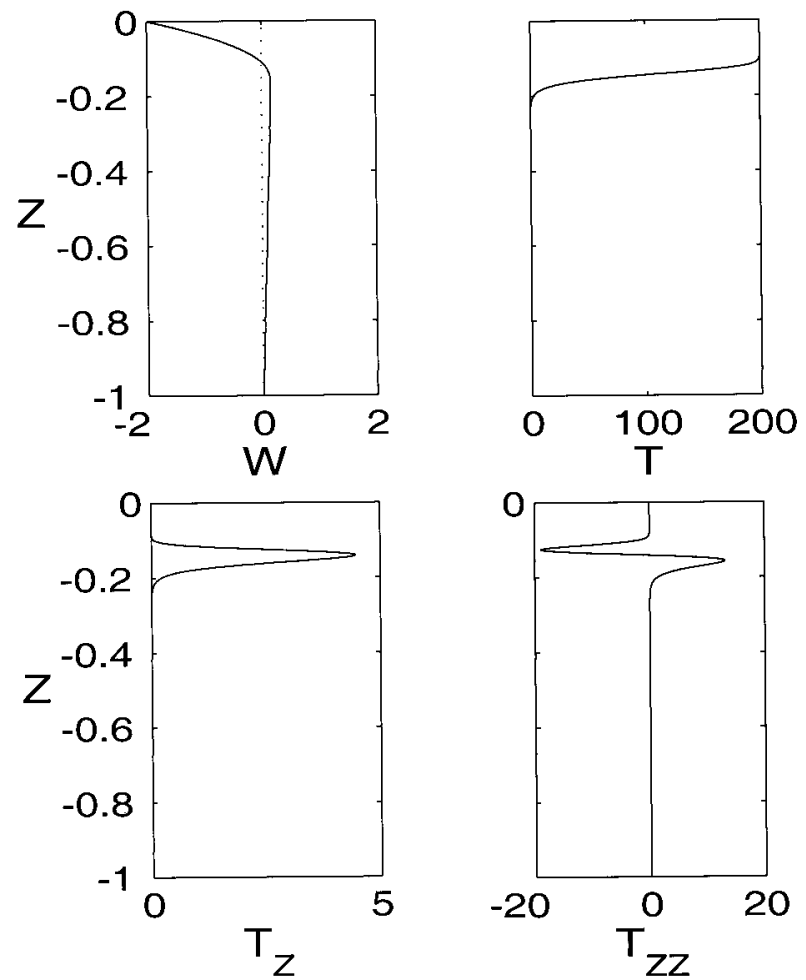

FIG. 7. Profiles of vertical velocity, temperature, $\partial T / \partial \zeta$, and $\partial^{2} T / \partial \zeta^{2}$ from solutions of the similarity equation (3.16), with imposed downward velocity at the top of the domain [i.e., $w(\zeta=1)=-2$ ].
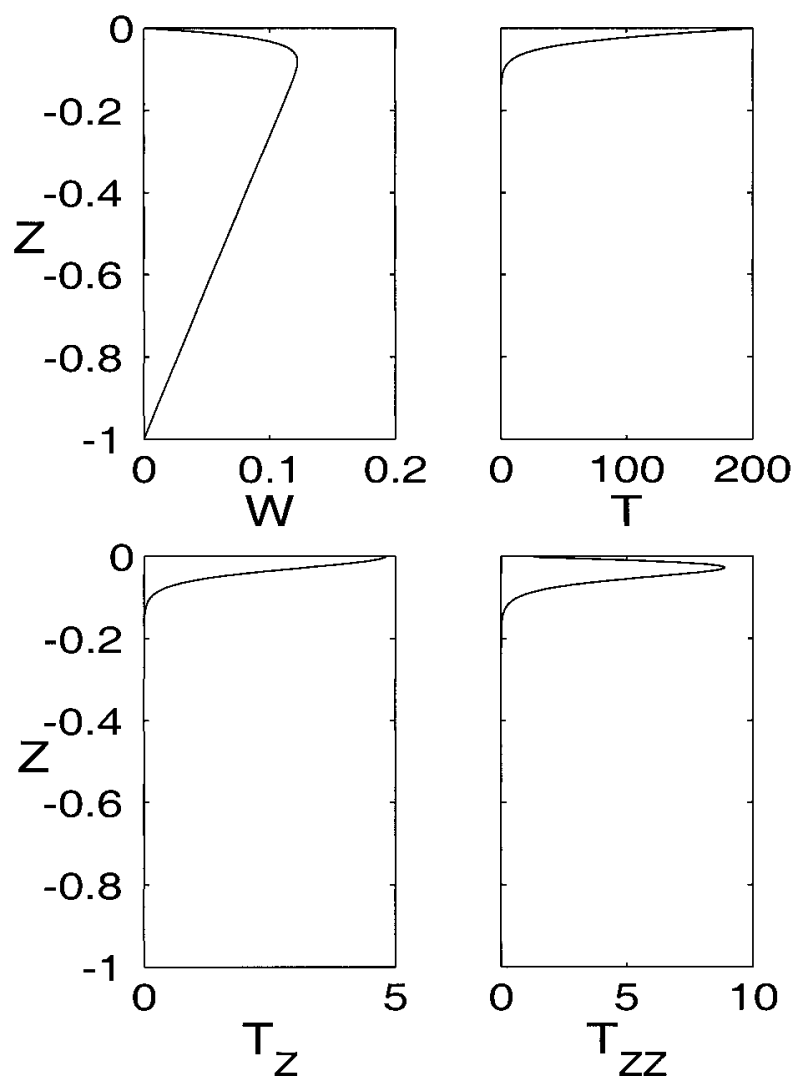

FIG. 8. Profiles of vertical velocity, temperature, $\partial T / \partial \zeta$, and $\partial^{2} T / \partial \zeta^{2}$ from solutions of the similarity equation (3.16), with zero vertical velocity at the top of the domain [i.e., $w(\zeta=1)=0$ ].

Figure 3 illustrates how the depth varies with the wind and is generally consistent with it being proportional to the square root of the amplitude of the wind.

At the base of the ventilated thermocline lies an internal boundary layer. If this obeys a form of advective diffusive balance,

$$
w \frac{\partial T}{\partial z} \approx k_{\nu} \frac{\partial^{2} T}{\partial z^{2}},
$$

then, as $k_{\nu} \rightarrow 0$ then (unless the vertical velocity decreases linearly or faster with $k_{v}$ ), the thickness of the thermocline will decrease, but the diffusive terms will remain finite. Adding only the diffusivity to the thermodynamic scaling, Eqs. (3.1)-(3.3) become

$$
\begin{aligned}
\frac{f V}{\delta} & \sim g \alpha \frac{\Delta T}{L} \\
\frac{V}{L} & \sim \frac{W}{\delta} \\
\frac{V \Delta T}{L}, \frac{W \Delta T}{\delta} & \sim \frac{k_{\nu} \Delta T}{\delta^{2}},
\end{aligned}
$$

where $\delta$ is to be interpreted as the thickness of the diffusive region and $W$ is no longer the prescribed Ek- 
(a)

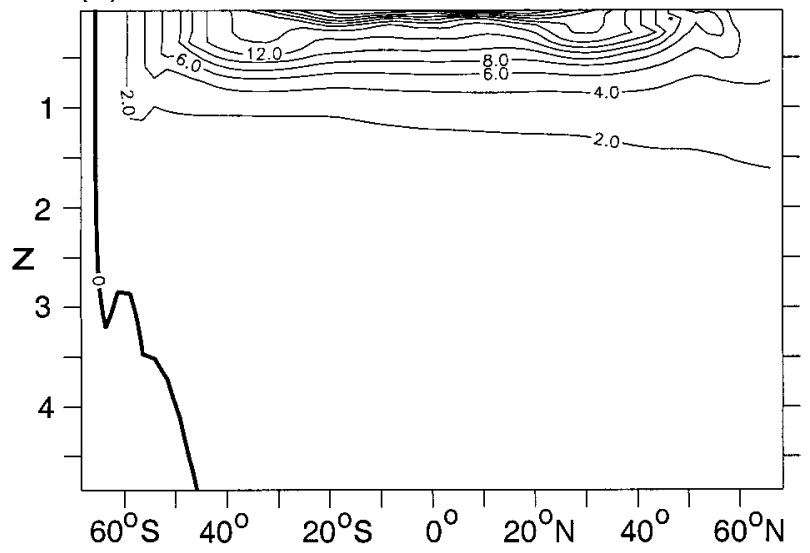

(c)

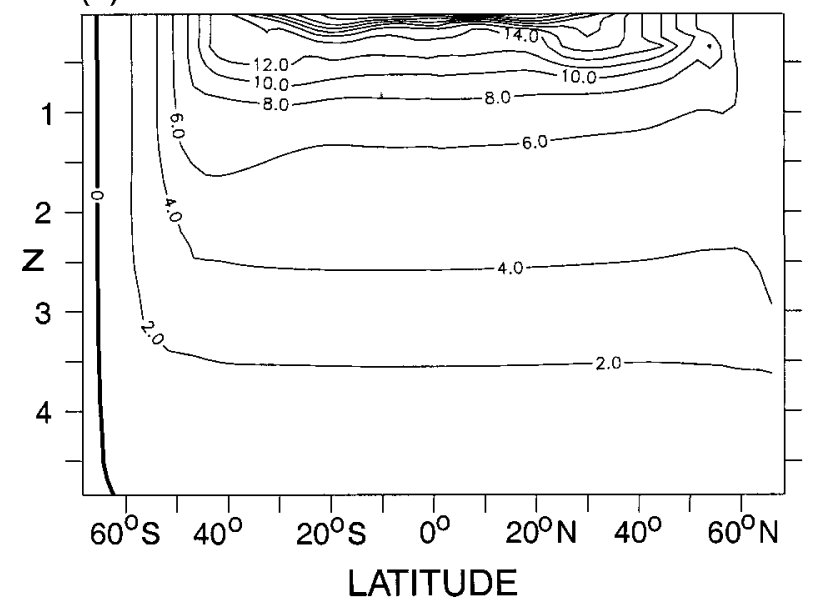

(b)

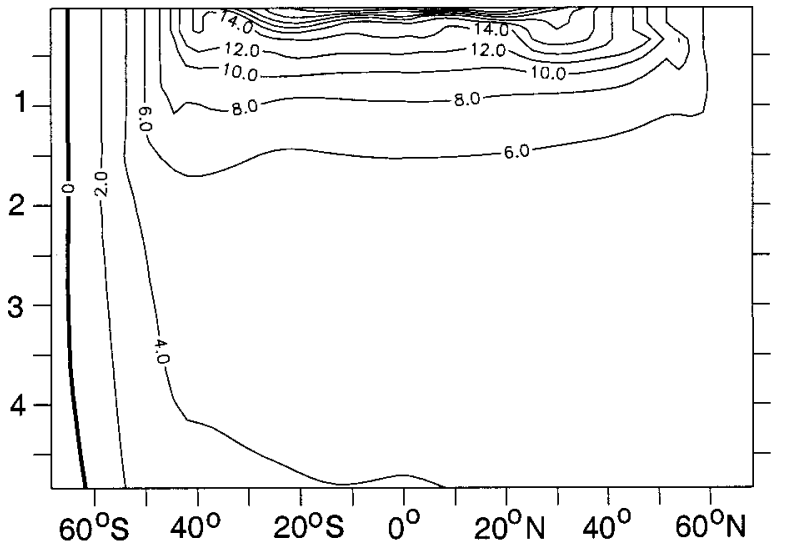

(d)

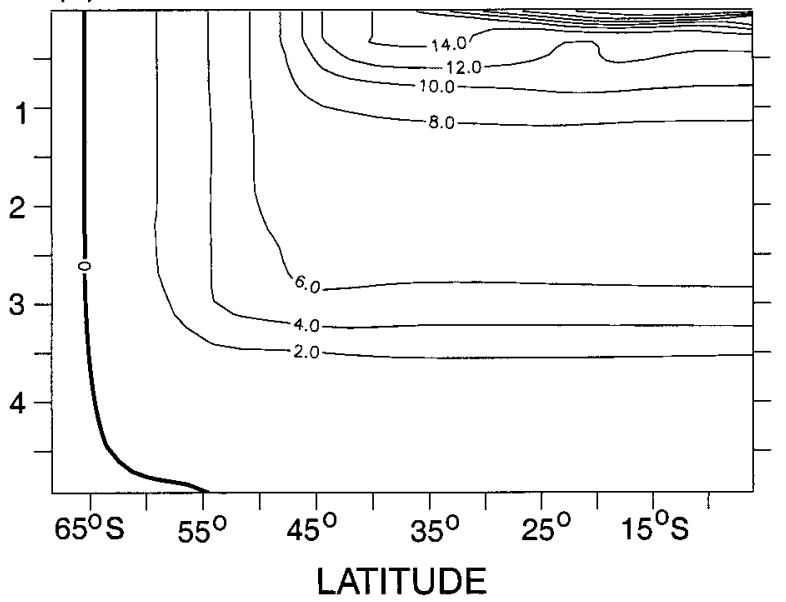

FIG. 9. Sections of temperature from four integrations that differ only in their geometry. (a) A closed, simply connected, flat-bottomed basin (G1). (b) A flat-bottomed basin closed everywhere except from $70^{\circ} \mathrm{S}$ to $50^{\circ} \mathrm{S}$; here the zonal boundary conditions are periodic (G2). (c) As for (b) but the opening is partially blocked by topography $2500 \mathrm{~m}$ high. Elsewhere the basin is flat bottomed (G3). (d) The same as (c) except the domain is confined to the Southern Hemisphere by a wall at the equator (G3s).

man pumping velocity but is the internally determined vertical velocity. We also assume that vertical temperature variations across the thermocline are comparable to the magnitude of horizontal temperature variations. These equations readily yield

$$
\begin{aligned}
\delta & \sim\left\{\frac{k_{\nu} f L^{2}}{g \alpha \Delta T}\right\}^{1 / 3} \propto k_{\nu}^{1 / 3} \\
W & \sim \frac{k_{\nu}}{\delta} \propto k_{\nu}^{2 / 3} .
\end{aligned}
$$

However, SV suggested that the horizontal scale should not be the gyre scale, but rather the horizontal distance across the sloping thermocline itself, $\hat{L}$ say. Referring to Fig. $2, \hat{L} / \delta=L / D$, where $D$ is an advective depth given by (3.4). Thus, (3.6) is replaced by

$$
\frac{f V}{\delta} \sim g \alpha \frac{\Delta T}{(\delta L / D)} .
$$

Using (3.4), (3.7), (3.8), and (3.11) yields

$$
\begin{aligned}
& \delta \sim\left\{k_{\nu}\left[\frac{f L^{2}}{g \alpha \Delta T W_{E}}\right]^{1 / 2}\right\}^{1 / 2} \propto k_{\nu}^{1 / 2} \\
& W \sim \frac{k_{\nu}}{\delta} \propto k_{\nu}^{1 / 2} .
\end{aligned}
$$

In this case we should expect the thermocline thickness to scale as $k_{\nu}^{1 / 2}$ provided that there is a wind stress to provide sloping isotherms. But, if there is no wind stress, the horizontal scale will be the scale of the domain and the thermocline thickness should scale as $k_{\nu}^{1 / 3}$. Figure 4 shows the results of two sequences of experiments to test this hypothesis. These sequences differ from each other only in that in one a wind stress was imposed, whereas in the other the wind is everywhere zero. For the purposes of these experiments, the thermocline thickness in the presence of wind was defined (as in SV) as the lower half-width at half-maximum of the $T_{z}$ peak. In the absence of winds, the thickness is the distance from the surface to the half-maxi- 

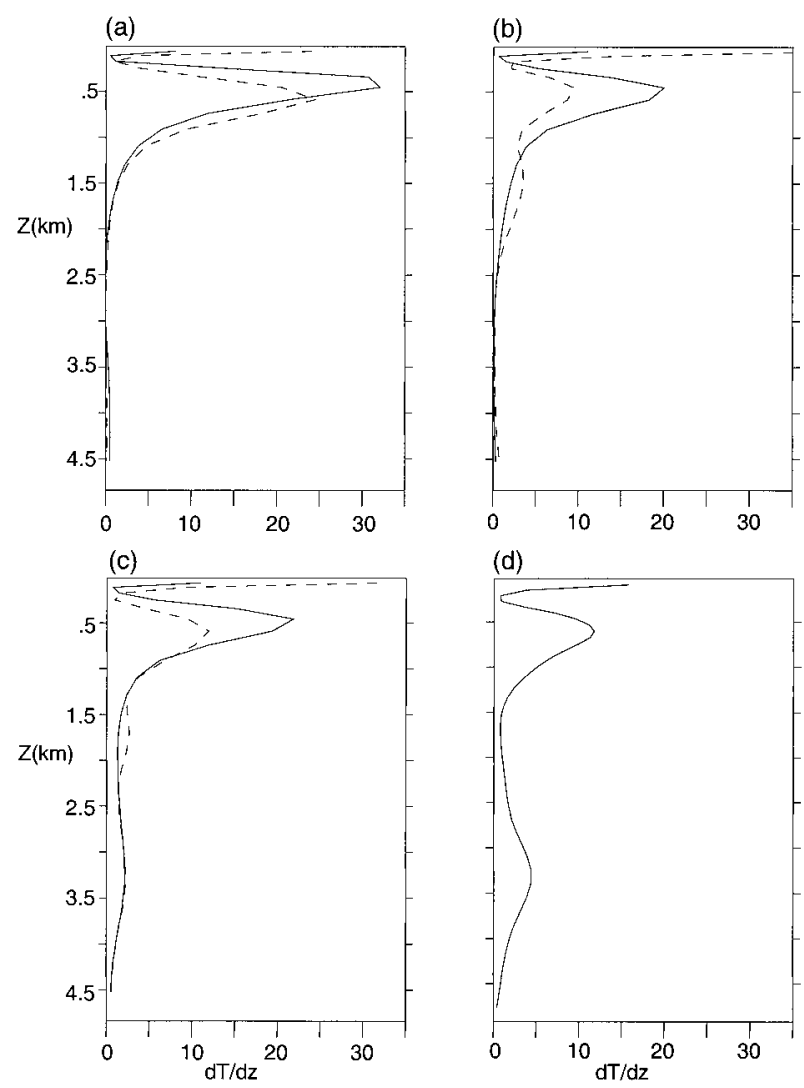

FIG. 10. Profiles of $\partial T / \partial z$ in the subtropical gyre in the integrations of Fig. 9. (a) A closed, simply connected, flat-bottomed basin (G1). (b) As in (a) but with an open channel at the southern end (G2). (c) Partially blocked channel (G3). (d) Same as (c) but Southern Hemisphere only (G3s). In (a), (b), and (c) the solid line is Northern Hemisphere and the dashed line is Southern Hemisphere.

mum of $T_{z}$. Although there is some subjectivity in the definitions themselves, they do provide an objective, if not unique, measure of thermocline thickness. For small diffusivity, the results show a clear difference in the scaling regimes with and without wind, in support of the scaling theory.

The strength of the meridional overturning circulation is consistent with these notions (Fig. 5), for small values of diffusivity. The overturning circulation is approximately proportional to $k_{\nu}^{1 / 2}$ and $k_{\nu}^{2 / 3}$ in the presence of and in the absence of Ekman forcing, respectively.

Finally, we performed a sequence of experiments in a closed, two-hemisphere domain (from $70^{\circ} \mathrm{S}$ to $70^{\circ} \mathrm{N}$ ). The surface forcing is again taken from observations, so the hemispheres are asymmetric. The results, with nonzero wind, approximately follow the internal boundary layer scaling for the internal thermocline (see Fig. 6). There are small differences in scaling between the two hemispheres, although the slope in both is close to $k_{v}^{1 / 2}$, perhaps slightly steeper. Perhaps more importantly, the qualitative two-thermocline picture still holds. This, and the differences between the one- and two-hemi- sphere general circulations, are discussed further in section 4.

\section{Crossover}

The estimates (3.4), (3.9), and (3.12) all coincide at a single value of the diffusivity given by

$$
k_{\nu} \sim W_{E}^{3 / 2}\left(\frac{f L^{2}}{g \alpha \Delta T}\right)^{1 / 2} .
$$

Physically, the diffusion is now sufficiently large enough for the diffusive thermocline to be as thick as the depth produced by Ekman pumping. For values of $k_{\nu}$ larger than this the diffusive scaling will dominate, whereas for smaller values the wind-effected scaling holds. For values of $\alpha=10^{-4} \mathrm{~K}^{-1}, f=10^{-4} \mathrm{~s}^{-1}, L=$ $5000 \mathrm{~km}, g=10^{3} \mathrm{~cm}^{2} \mathrm{~s}^{-1}, \Delta T=20 \mathrm{~K}$, and $W_{E}=10^{-4}$ $\mathrm{cm} \mathrm{s}^{-1}$ the critical value for $k_{\nu}$ is $2 \mathrm{~cm}^{2} \mathrm{~s}^{-1}$.

An equivalent estimate, but one that may be more useful from an observational point of view, is

$$
k_{\nu} \sim W_{E} D \sim \frac{V D^{2}}{L},
$$

where $D$ is the depth or thickness (the same in this limit) of the thermocline. This estimate is simply that which arises by equating the Ekman pumping velocity with the vertical velocity associated with the meridional overturning. Taking $D=500 \mathrm{~m}$ gives the somewhat higher estimate for the cross-over value for $k_{\nu}$ to be $5 \mathrm{~cm}^{2} \mathrm{~s}^{-1}$. These are only scaling estimates and, as such, may be wrong by nondimensional constants, but nevertheless they are all well above typical measured values of the diffusivity in the upper ocean, indicating the importance of wind-driven processes in thermocline and thermohaline circulation scaling, and consistent with the existence of mode water in subtropical gyres. To test this we have performed integrations over a large range of diffusivities; Fig. 5 shows how the meridional overturning circulation varies as a function of diffusivity. The results with Ekman forcing are consistent with two scaling regimes: at small values of $k_{\nu}$ the meridional overturning circulation scales as the one-half power of diffusivity and at larger values as the two-thirds power. The cross-over appears to occur at rather smaller values of the diffusivity than the estimates given above, but these are scaling estimates and therefore are not necessarily quantitatively accurate. The results with zero wind forcing show no evidence of two scaling regimes.

\section{c. Similarity theory}

Stommel and Webster (1962) proposed a nonlinear ordinary equation that models some of the properties of the thermocline equations. A family of related equations was subsequently derived more systematically by Young and Ierley (1986) using similarity theory. The simplest, 
(a)

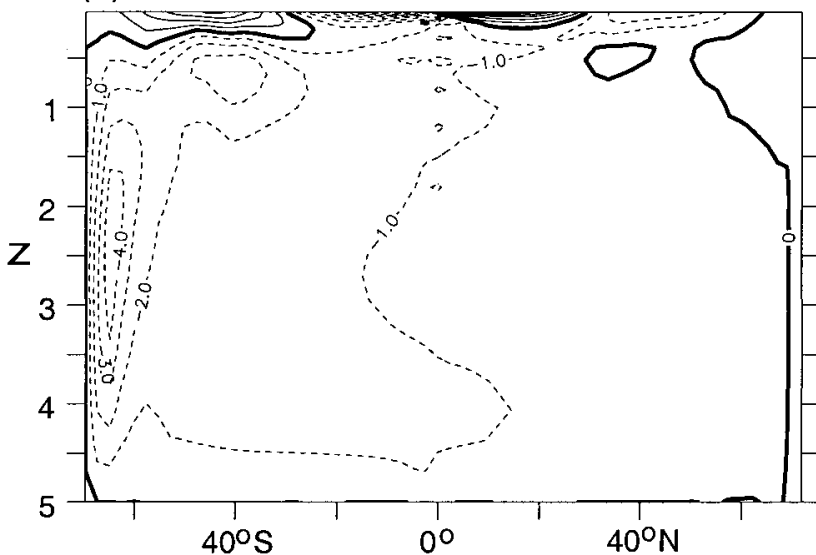

(c)

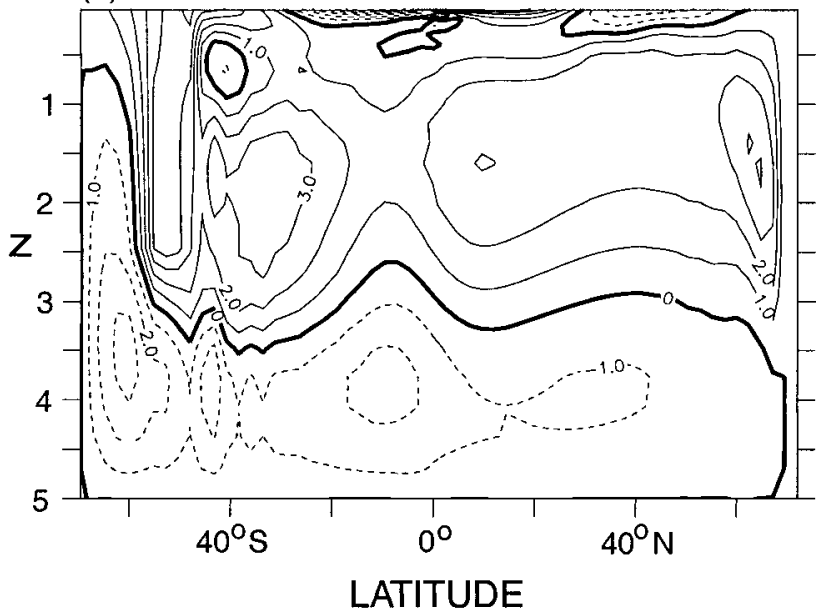

(b)

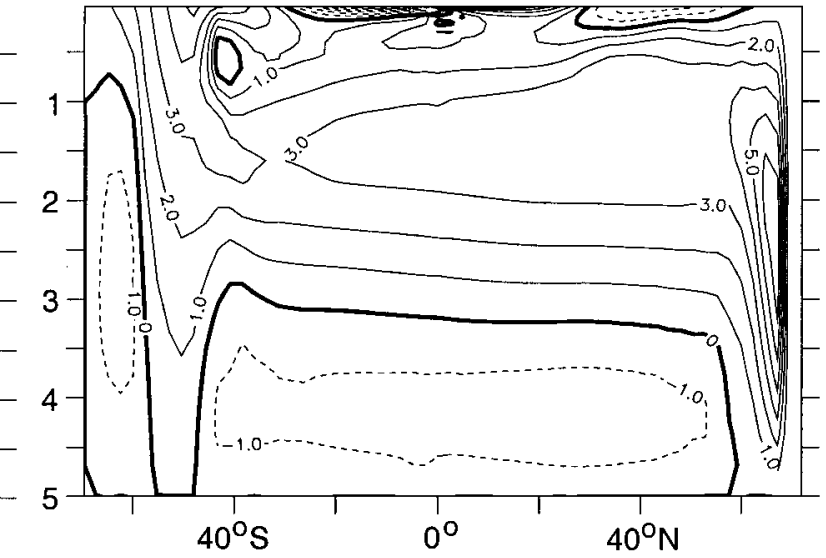

(d)

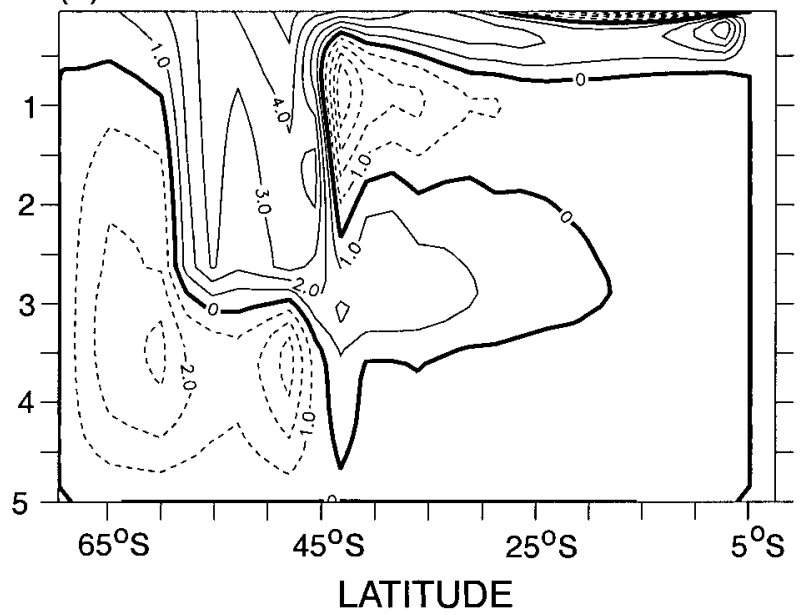

FIG. 11. Meridional overturning circulation (units of $10^{12} \mathrm{~cm}^{3} \mathrm{~s}^{-1}$ ) from the integrations of Fig. 9. (a) A closed, simply connected, flatbottomed basin (G1). (b) As in (a) but with an open channel at the southern end (G2). (c) Partially blocked channel (G3). (d) As for (c) but Southern Hemisphere only (G3s). Solid (dashed) lines mostly correspond to clockwise (anticlockwise) circulation.

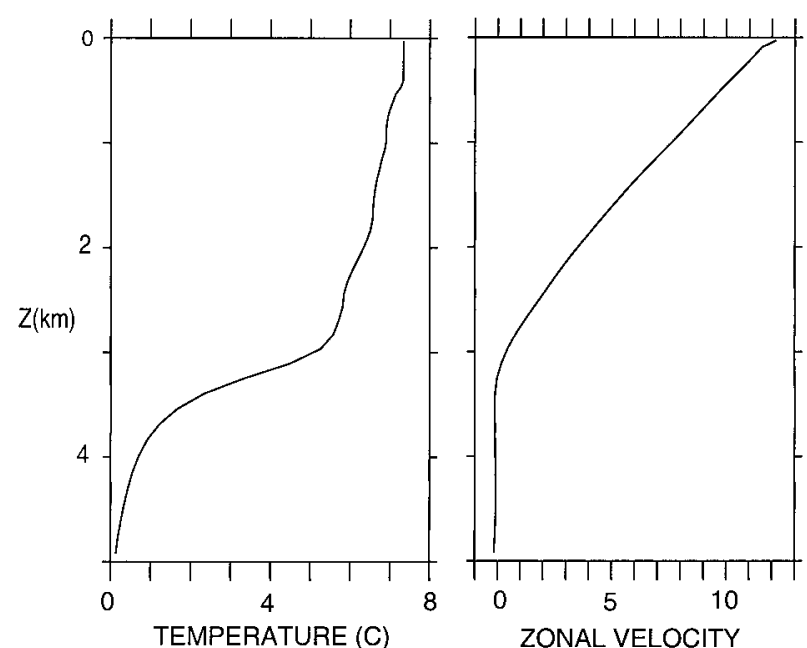

FIG. 12. Profiles of temperature and zonal velocity in the center of the circumpolar channel, in G3s. and arguably the most physical, member of Young and Ierley's family is

$$
\left\{2 \tilde{W}-\zeta \frac{d \tilde{W}}{d \zeta}\right\} \frac{d^{3} \tilde{W}}{d \zeta^{3}}=k_{\nu} \frac{d^{4} \tilde{W}}{d \zeta^{4}},
$$

with $T \sim d^{2} \tilde{W} / d \zeta^{2}$. Some of the properties of this equation were also discussed by SV. It is interesting to ask whether the equation can also reproduce the two scaling regimes, with and without an imposed downward vertical velocity at the top of the one-dimensional domain. Figures 7 and 8 show profiles of $\partial T / \partial \zeta$ and vertical velocity from numerically obtained solutions of the equation. Solutions were obtained using Newton's method, with 400 equally spaced grid increments. Test solutions at still higher resolution indicated that numerical convergence was obtained. Solutions with an imposed Ekman pumping are obtained by imposing boundary conditions of

$$
W(\zeta=0)=0 \quad W(\zeta=1)=W_{E},
$$


(where $W_{E}$ is the given vertical velocity at the top) as well as $\partial T / \partial z=0$ at $\zeta=0,1$. A thin internal boundary layer (i.e., a thermocline) forms where the vertical velocity is zero, and the thickness of the thermocline may be defined, fairly unambiguously, as the distance between the extrema in $\partial^{2} T / \partial \zeta^{2}$ on either side of this thermocline. Solutions with no imposed Ekman forcing are obtained by setting $W(\zeta=1)=0$. The thermocline is now adjacent to the upper boundary, forming a more conventional boundary layer. Its thickness may be defined as the distance from the top to the first extrema in $\partial^{2} T / \partial \zeta^{2}$. Results both with and without Ekman pumping are shown in Figs. 7 and 8, and, as for the primitive equation ocean model, two scaling regimes are apparent (Fig. 4b). With Ekman pumping the internal thermocline scaling holds, namely that the thickness is proportional to $k_{\nu}^{1 / 2}$ and the vertical velocity is proportional to $k_{\nu}^{1 / 2}$. In the absence of wind forcing the thermocline thickness scales as $k_{\nu}^{1 / 3}$, with a corresponding maximum vertical velocity proportional to $k_{v}^{2 / 3}$. If $\zeta=0$ in (3.16) the equation is similar to that analyzed by Salmon (1990), and analysis of that equation indicates that the boundary layer thickness should scale as $k_{\nu}^{1 / 3}$; this is confirmed by numerical solution (not shown here), both in the presence and absence of wind. The agreement of the PE model results, the similarity theory, and the simple scaling theory indicates that a form of advective-diffusive balance is likely to hold at the base of the main thermocline and that the essence of the two scaling regimes is captured by the scaling argument.

\section{Geometric effects on stratification}

\section{a. A Southern Hemisphere channel}

One feature of the above simulations that corresponds particularly poorly with the structure of the observed ocean is the absence of deep stratification. This is not uncommon in such idealized simulations and the importance of vertical (or diapycnal) diffusion was noted by, among others, Cummins et al. (1990), Cummins (1991), SV, and Samelson (1998). Cummins et al. (1990) and Samelson (1998) further explored the effects of the diffusivity on the deep stratification. Samelson found that, in a simply connected, single-hemisphere basin, in order to produce a deep stratification comparable to observed values a locally large diffusivity $\left(\sim 100 \mathrm{~cm}^{2} \mathrm{~s}^{-1}\right)$ over a broad boundary region was required. Observational measurements of the diffusivity are not conclusive, and certainly the possibility of locally large diffusivities cannot be ruled out (e.g., Polzin et al. 1997) in spite of seemingly small diffusivities in the more open ocean away from distinctive topographic features (Gregg 1987; Ledwell et al. 1993; Toole et al. 1994). Nevertheless, the ambiguity of the measurements suggests that it might be useful to explore whether other sources of deep stratification might exist; for example, the results of Cox (1989) and Toggweiler and Samuels
TABLE 1. Brief description of experiments with varying geometry.

\begin{tabular}{|c|c|}
\hline Experiment & Geometry \\
\hline G1 & Single, enclosed flat-bottomed basin: $70^{\circ} \mathrm{N}-70^{\circ} \mathrm{S}$. \\
\hline G2 & As for $\mathrm{G} 1$ but zonally periodic from $45^{\circ} \mathrm{S}$ to $70^{\circ} \mathrm{S}$. \\
\hline G3 & $\begin{array}{l}\text { As for } \mathrm{G} 2 \text { but with a meridional ridge, } 2500 \mathrm{~m} \text { high, } \\
\text { partially blocking the periodic channel. }\end{array}$ \\
\hline G3s & $\begin{array}{l}\text { As for G3 by the domain is confined to the Southern } \\
\text { Hemisphere. }\end{array}$ \\
\hline G4 & $\begin{array}{l}\text { As for } \mathrm{G} 3 \text { but ridge is lower ( } 1500 \mathrm{~m} \text { high, i.e., top } \\
3500 \text { below surface). }\end{array}$ \\
\hline G5 & $\begin{array}{l}\text { As for } \mathrm{G} 3 \text { but ridge is higher ( } 3000 \mathrm{~m} \text { high, i.e., top } \\
2000 \mathrm{~m} \text { below surface). }\end{array}$ \\
\hline G6s & $\begin{array}{l}\text { As for } \mathrm{G} 3 \mathrm{~s} \text { but the periodic channel is wider, i.e., of } \\
\text { greater meridional extent, from } 30^{\circ} \mathrm{S} \text { to } 70^{\circ} \mathrm{C} \text {. }\end{array}$ \\
\hline G7s & $\begin{array}{l}\text { As for } \mathrm{G} 3 \text { but the periodic channel is narrower, from } \\
55^{\circ} \mathrm{S} \text { to } 70^{\circ} \mathrm{S} \text {. }\end{array}$ \\
\hline G8 & $\begin{array}{l}\text { As for G3 but with zero surface temperature gradient } \\
\text { across the periodic channel. }\end{array}$ \\
\hline G9 & $\begin{array}{l}\text { As for } \mathrm{G} 8 \text { but the temperature difference across the } \\
\text { closed domain is larger, equalling the temperature } \\
\text { difference of } \mathrm{G} 3 \text { across its entire hemisphere. }\end{array}$ \\
\hline G10 & $\begin{array}{l}\text { As for G3 except that the periodic channel is in the } \\
\text { warmer (Northern) hemisphere. }\end{array}$ \\
\hline G10n & $\begin{array}{l}\text { As for G10 except that the domain is confined to the } \\
\text { Northern Hemisphere. }\end{array}$ \\
\hline G11 & $\begin{array}{l}\text { As for G3 but with reduced wind over the channel } \\
\text { (see Fig. 22). }\end{array}$ \\
\hline G12 & As for G11 but with Zero wind over the channel. \\
\hline
\end{tabular}

(1995) further suggest that the presence of an Antarctic Circumpolar Current exerts a significant influence on the meridional overturning circulation. Observational syntheses of water masses and generalized "conveyor belt" descriptions of the general circulation indicate a flow with complex topology (Schmitz 1995), but in which (at least in the Atlantic) the presence of Antarctic Bottom Water underneath North Atlantic Deep Water may be responsible for a significant fraction of the deep stratification.

A number of idealized experiments are reported that explore the production of deep stratification (see Table 1). The first three illustrate in the most basic form the profound effects of model geometry on the production of abyssal stratification. In experiment G1 the model is configured as a single, closed basin, extending from $70^{\circ} \mathrm{N}$ to $70^{\circ} \mathrm{S}$, with a longitudinal extent of $64^{\circ}$ (this is the same geometry that produced the results of Fig. 6). The model is flat-bottomed everywhere with a depth of $5000 \mathrm{~m}$. In the second experiment, G2, the boundary conditions for latitudes $45^{\circ}-70^{\circ}$ are zonally periodic, rather than closed. That is, a "channel" is opened at the southern end of the domain, but the model remains flat-bottomed everywhere. In the third, G3, a thin topographic ridge extends meridionally across the channel opening to a height of $2500 \mathrm{~m}$. All other aspects of the experiments are the same as before. Experiment G3s is the same as G3, save that a wall is placed at the equator and the model is confined to the Southern Hemisphere. The surface buoyancy forcing is such that the densest water forms at the southern end of the domain in all cases. The experiments are each spun up from rest to 
(a)

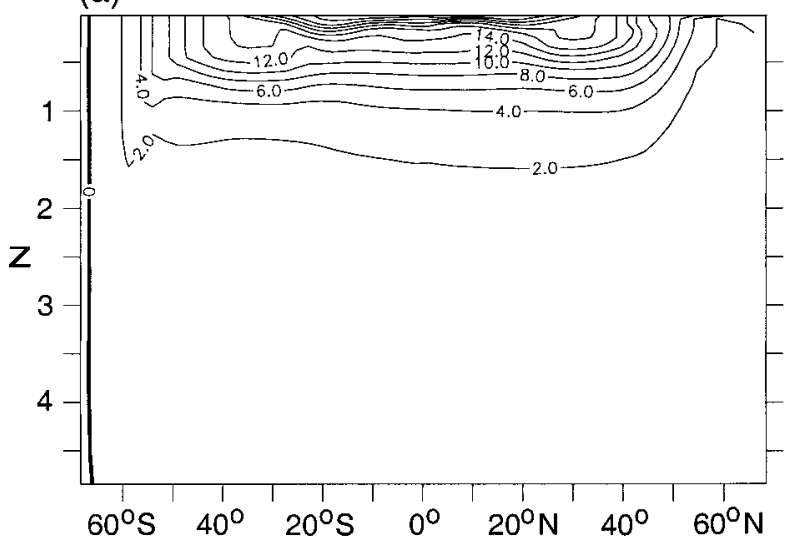

(b)

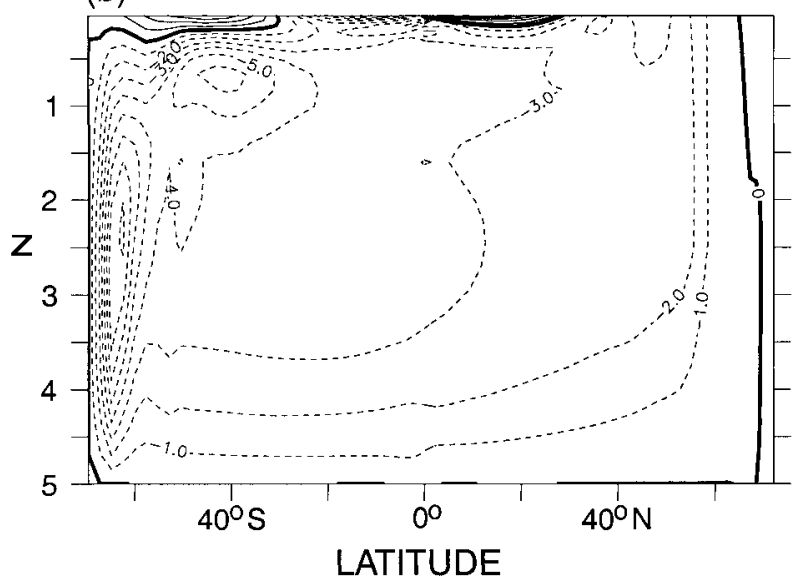

FIG. 13. Temperature section (a) and meridional overturning circulation (b) from integration G10 - a two-hemisphere integration, with partially open Northern Hemisphere channel.

equilibrium over about 5000 years, with gradually decreasing diffusivity, the final equilibration interval of about 2000 years having a uniform diffusivity of 0.1 $\mathrm{cm}^{2} \mathrm{~s}^{-1}$. The configuration of some of these experiments is similar to some of those of Gill and Bryan (1971) and Cox (1989), although for simplicity we set the channel flush against the southern boundary.

The temperature structure of the final states is illustrated in Figs. 9 and 10. In all cases the upper ocean stratification is qualitatively similar. There are, as for the single-hemisphere integrations, two upper-ocean maxima in the stratification $(\partial T / \partial z)$, one near the surface corresponding to a ventilated thermocline and a second, internal maximum at about $500 \mathrm{~m}$. In all cases the uppermost maxima (a measure of the strength of the ventilated thermocline) is strongest in the Southern Hemisphere, consistent with the stronger Southern Hemisphere winds; it is also stronger in the two cases with a channel because the lack of zonal boundaries leads to a stronger near-surface flow. The deep stratification and the meridional overturning circulation qualitatively differs between the various cases. In the closed domain the (a)

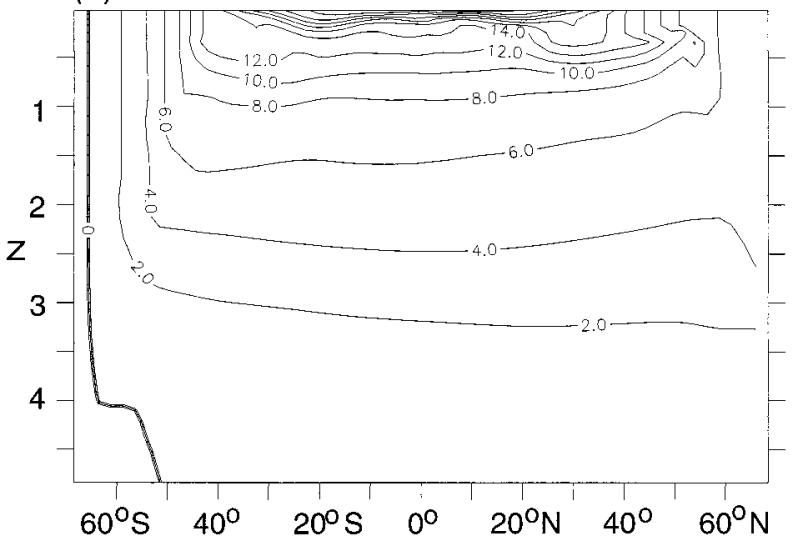

(b)

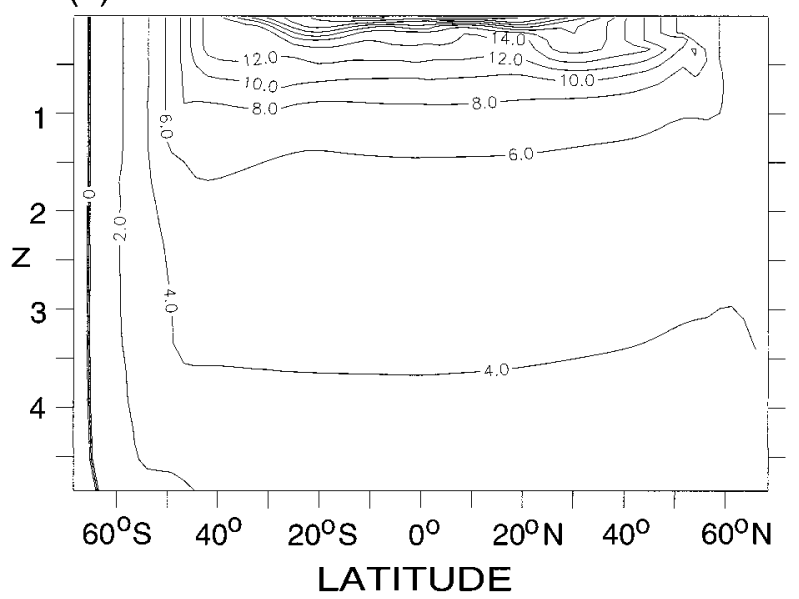

FIG. 14. Temperature sections from two integrations differing only in the height of the ridge in the periodic channel (a) from G5 (ridge is $3500 \mathrm{~m} \mathrm{high}$ ) and (b) from G4 (ridge is $1500 \mathrm{~m} \mathrm{high}$ ).

abyss largely fills with the densest available water, in this case from the southern end of the domain. The meridional overturning circulation giving rise to this is strongly asymmetric (Fig. 11); downwelling is confined to the southern edge of the model ocean, with the strongest upwelling just equatorward of this and generally weak, fairly uniform upwelling over most of the rest of the domain. If the southern part of the domain is an open channel, then the geostrophic constraint does not allow efficient equatorward spreading of the dense water: If the balance in the zonal momentum equation is (in Cartesian form)

$$
f v=\frac{1}{\rho_{0}} \frac{\partial p}{\partial x}+F,
$$

where $F$ represents small frictional and nonlinear terms, then with zonally periodic boundary conditions

$$
f \bar{\nu}=\bar{F} \text {. }
$$

Thus, on the zonal average, meridional spreading of polar waters is due only to nongeostrophic effects and 
(a)

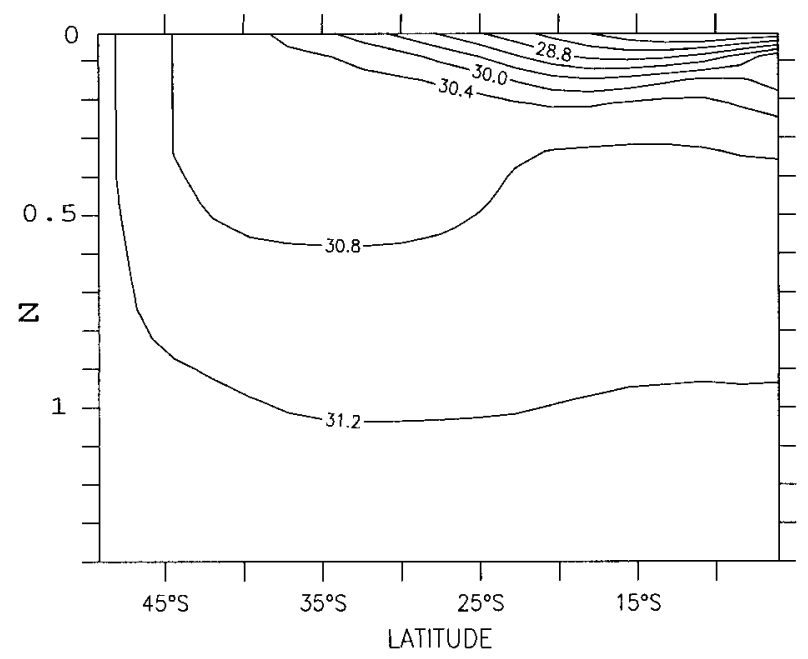

(c)

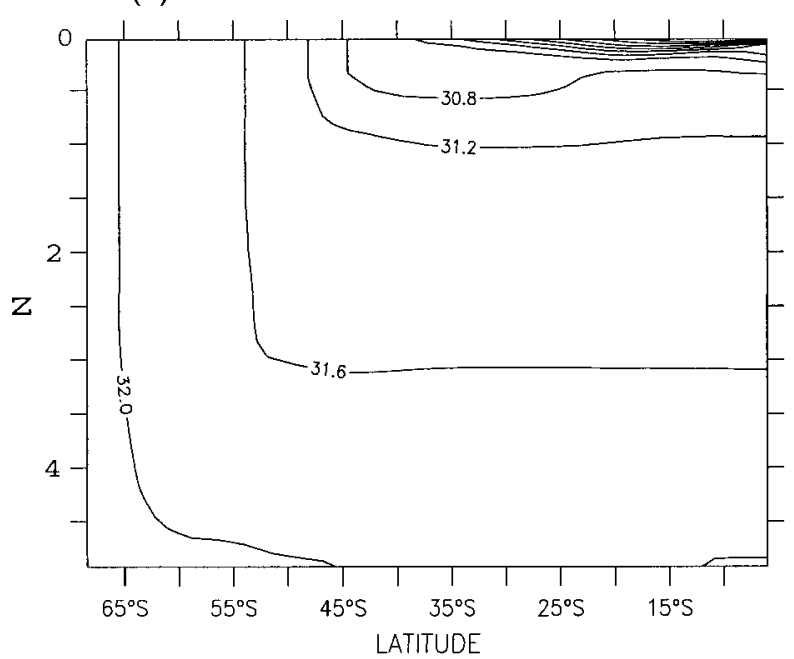

(b)

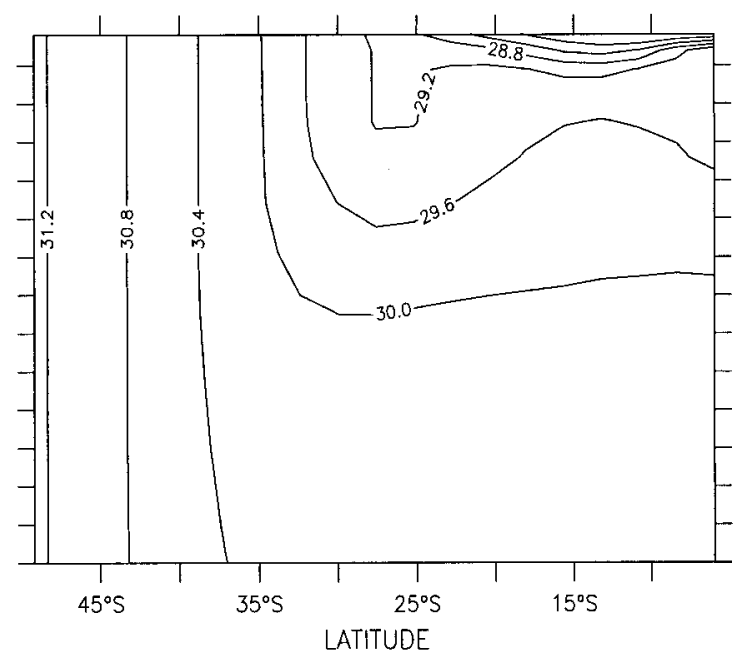

(d)

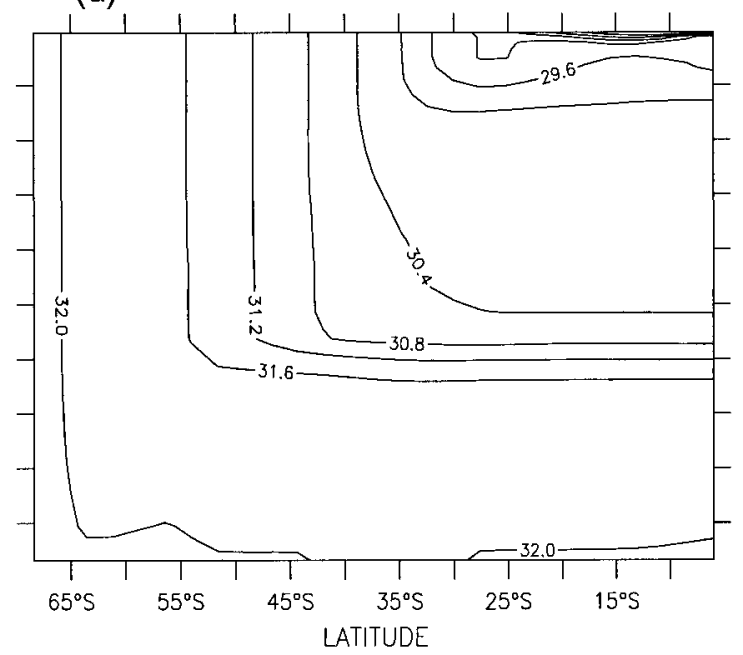

FIG. 15. Density sections from two integrations differing only in the width of the the periodic channel. The left panels are from G3s; the right panels are from G6s-channel is wider, moving equatorward well beyond the latitude of zero wind curl. The upper panels show the upper $1.5 \mathrm{~km}$ of the domain, the lower panels the full depth.

is perforce very weak, in the absence of eddies. This is reflected in the results with a completely open channel (G2), shown in Fig. 9b. The abyss is still very homogeneous, but now it is full of water that has come from the warmer northern ocean; the dense southern water can barely spread beyond the latitude of the channel opening. Consistently, the meridional overturning circulation (Fig. 11b) shows the strongest cell at the northern edge of the domain. (Although it cannot be ruled out that an extended integration of the model would allow further spreading of Southern Hemisphere bottom water into the Northern Hemisphere and deep stratification, this process is extremely slow; a time period of tens of thousands of years would be required to see any noticeable difference from Fig. 9b.)
A partially blocked channel is different again. Now a zonal pressure gradient, and hence an associated meridional flow, can exist but only for deep water, below the level of the ridge. This is reflected in the meridional overturning pattern in (Figs. 11c and 11d). The temperature in the channel is virtually uniform below the sill (Fig. 12a), but is almost equal to that at the surface above - that is, the isotherms are almost vertical to the level of the sill. The zonal velocity is then reflective of thermal wind balance (Fig. 12b), increasing almost linearly above the sill. The surface zonal velocity in the channel is thus reflective of the meridional temperature gradient across it, rather than the wind forcing itself [and as in the analytic model of Samelson (1999)]. "Bottom water" is then produced primarily from the 

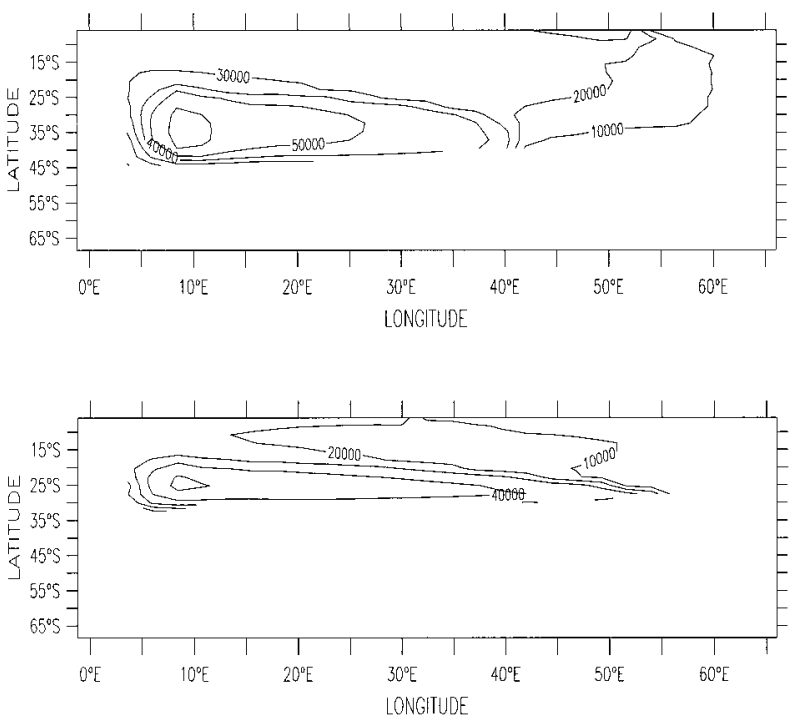

FIG. 16. Contours of depth of the lowest density layer of the ventilated thermocline for the same integrations as in Fig. 15, that is, G3 and G6s.

densest water in the channel, and this can spread well into the opposite hemisphere, remaining confined below the height of the topographic ridge, producing a thin layer of deep stratification at its top.

\section{b. Circulation in one and two hemisphere cases}

Consider the simpler single hemisphere simulation (G3s) first. There is a very well defined "abyssal thermocline" that separates deep bottom water from the "intermediate water" above (e.g., Fig. 9d). The temperature difference across this is approximately equal to the surface temperature difference across the periodic channel. Above the abyssal thermocline lies a second meridional overturning cell that forms another thermostad of intermediate water, the temperature of which is approximately that of the surface temperature at a latitude just poleward of the equatorial edge of the channel. Surface water equatorward of this is entrained into the main thermocline, some being entrained into the internal thermocline and water in the subtropical gyre being subducted into a ventilated thermocline. The temperature difference across the main thermocline (internal plus ventilated) is approximately equal to the surface temperature between the equator and the equatorial edge of the circumpolar channel, which is the approximate latitude of formation of intermediate water; thus, it is much weaker than in the case with no channel since, in that case, the main thermocline is a mapping of the entire pole-equator temperature gradient. In the case with a blocked channel, the abyss contains two types of water and so takes up some of this stratification, which is thus much higher than in the fully closed (G1) or fully open (G2) cases.

In the two-hemisphere simulation (G3) the flow is

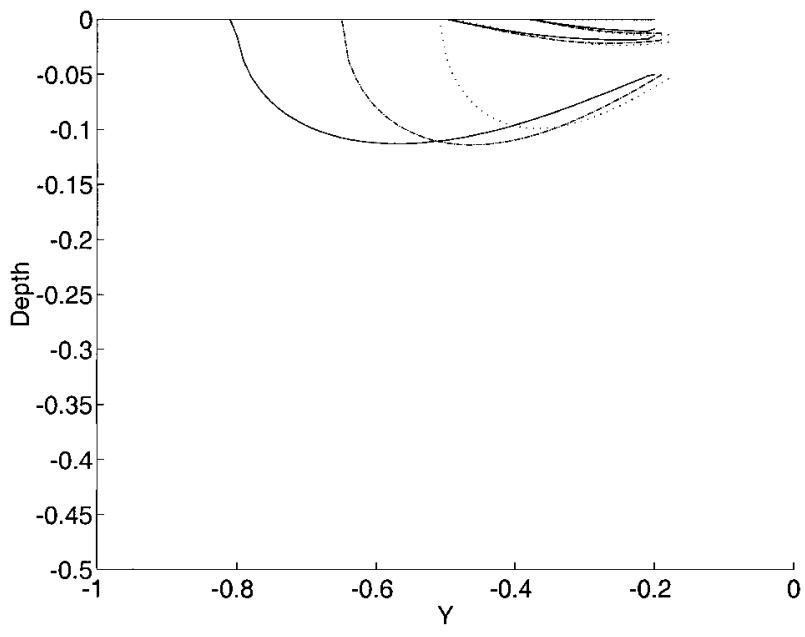

FIG. 17. Sections of layer depth from three three-layer analytic thermocline calculations, in the region of Ekman downwelling. The calculations differ primarily in the latitude of zero wind curl. The outcrop latitudes and wind stress at low latitude are taken from the PE simulation.

complicated by another source of dense water at high northern latitudes. The importance of this water is largely determined by its density relative to the Southern Hemisphere, and there are three cases to consider. In the first case, the water in the opposing hemisphere is
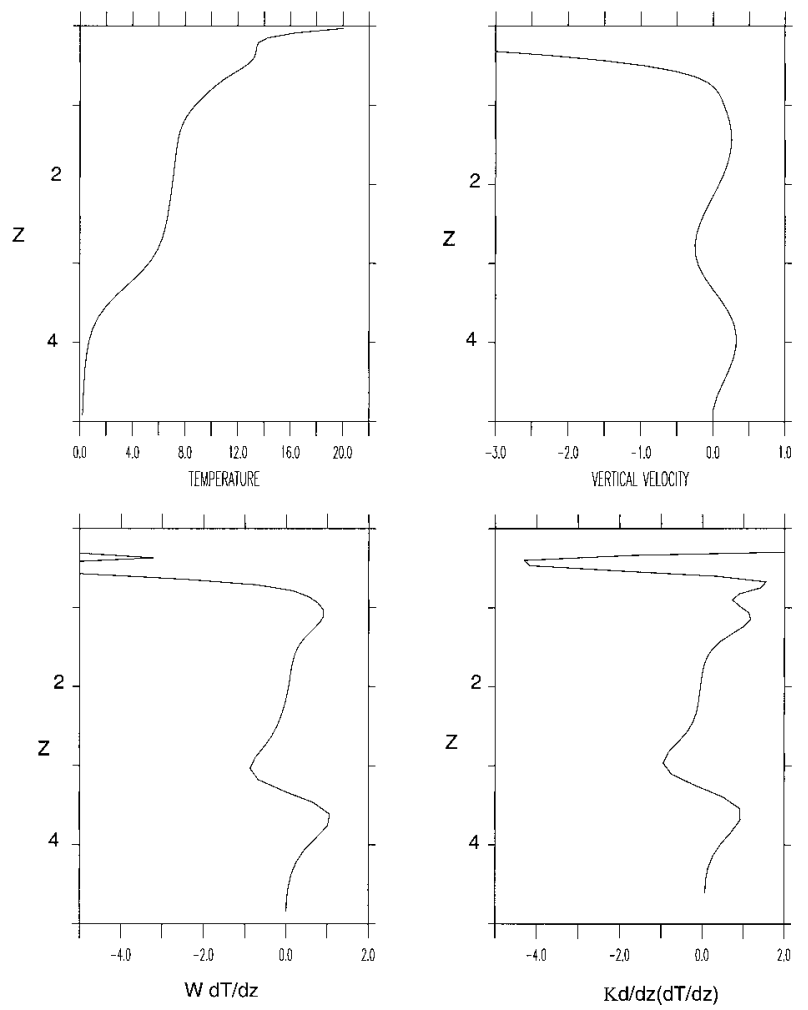

FIG. 18. Profiles of temperature, vertical velocity, $w \partial T / \partial z$, and $\partial^{2} T / \partial z^{2}$ in the center of the model domain, equatorward of the model circumpolar channel. 


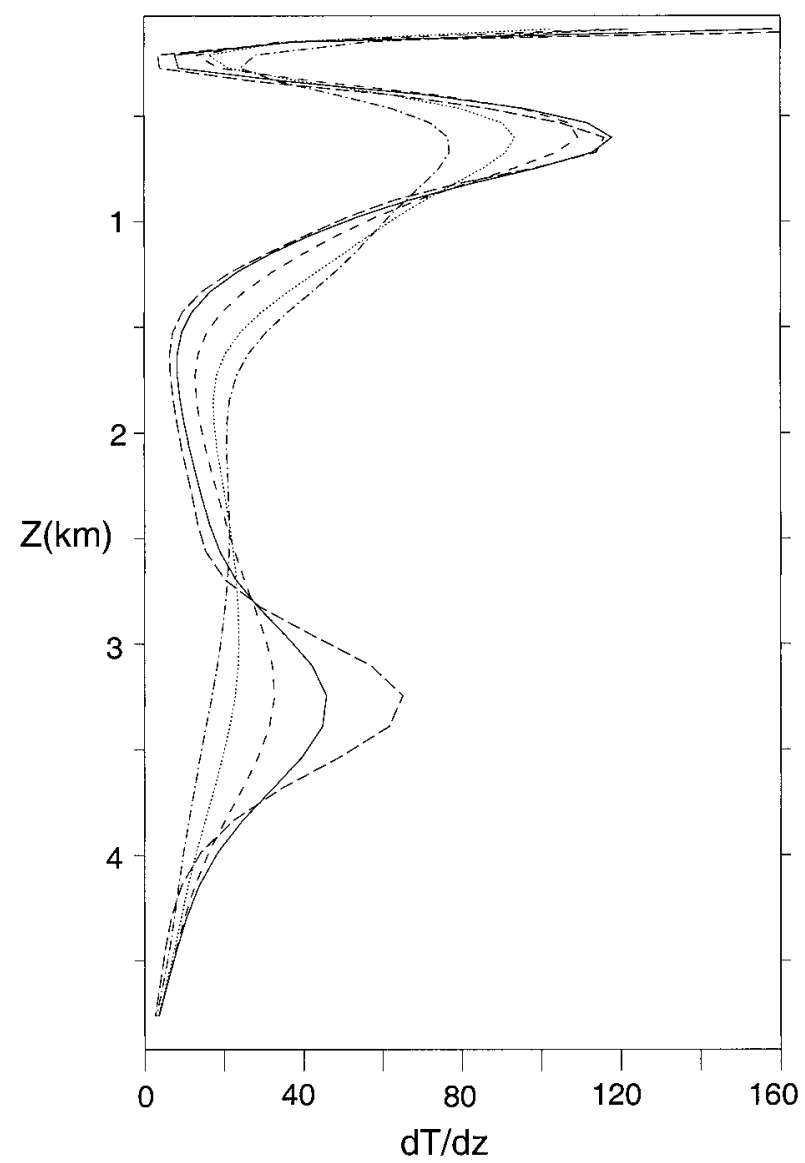

FIG. 19. Profiles of vertical derivative of temperature, for five different values of diffusivity $\left(1.2,0.8,0.4,0.2\right.$, and $\left.0.1 \mathrm{~cm}^{2} \mathrm{~s}^{-1}\right)$ at $35^{\circ} \mathrm{S}$, in G14. As diffusivity falls, both the main internal thermocline and the abyssal thermocline become thinner and sharper, but the ventilated thermocline is virtually unaltered.

denser than any produced in the hemisphere with the channel; the abyss then completely fills with that water and virtually no deep stratification is produced. This is equivalent to experiment G10 (illustrated in Fig. 13). (Here we have simply moved the channel to the Northern Hemisphere, keeping the surface boundary conditions the same.) The dense high-latitude colder (southern) hemisphere waters are free to spread, since this hemisphere is completely enclosed, and the abyss fills with these waters, becoming a homogeneous mass with little stratification. Although the partially blocked channel would mechanically allow the spreading of polar water from the warmer hemisphere, the water is insufficiently dense to do so; the meridional overturning becomes dominated by a single cell, of largest intensity in the Southern Hemisphere. If in this case with a Northern Hemisphere channel the domain is confined to the Northern Hemisphere, then naturally this hemisphere does become stratified.

The second case is that in which on the water from the opposing hemisphere is less dense than the water at the equatorial end of the channel. Then, Southern Hemi-

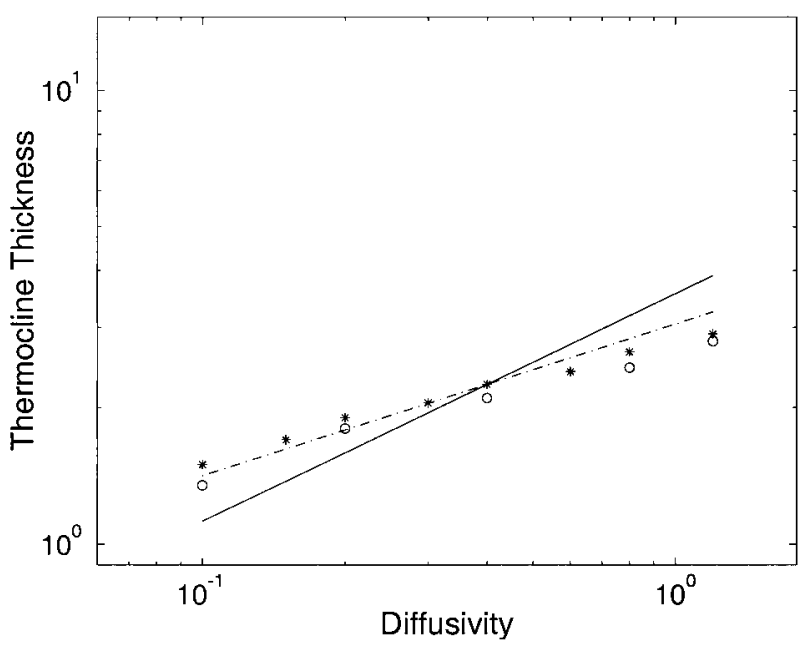

FIG. 20. Thickness of the abyssal thermocline as a function of vertical diffusivity $k_{\nu}$. The solid line is proportional to $k_{\nu}^{1 / 2}$ and the dashed line proportional to $k_{v}^{1 / 3}$. The stars are results from G10n, the open circles are results from $\mathrm{G} 3 \mathrm{~s}$.

sphere intermediate water is denser than any potential Northern Hemisphere deep water, and formation of the latter is suppressed. It then has little influence on the circulation in that hemisphere, and the Southern Hemisphere circulation is essentially that of the single hemisphere integration G3s and no deep Northern Hemisphere water ("NADW") is produced.

The third and intermediate case, and that which corresponds most to the real ocean, is one in which the density of the Northern Hemisphere water is the same as that of water somewhere in the Southern Hemisphere channel; that is, its density is between that of Southern Hemisphere bottom and intermediate water. The Northern Hemisphere water then serves to "split" the abyssal thermocline, and the cell of Southern Hemisphere intermediate water is much shallower, and partially entrained into the main thermocline-note the different depth of the $6^{\circ}$ isoline in Figs. 9c and 9d although the outcrop latitude is virtually the same in both cases. Note too the less well defined abyssal thermocline in the twohemisphere case and the different meridional overturning circulation. In the single hemisphere case the meridional overturning cell of intermediate water fills the domain between the abyssal thermocline and the main thermocline, whereas in the two-hemisphere case the cell of intermediate water is rather shallow, trapped between the main thermocline and the Northern Hemisphere deep water. As a consequence, the meridional heat transport in the Northern Hemisphere is much larger in the two cases with an open Southern Hemisphere channel because of the greater dominance of the Northern Hemisphere meridional overturning cell.

The abyssal flow is no longer uniformly poleward in either G3 or G3s; it is a rather complicated baroclinic structure, and no longer a broad, fairly uniform upwelling accompanied by a poleward motion, as in the 
enclosed single-basin integrations and in the framework of Stommel and Arons (1960).

\section{c. Effects of ridge height and width, and the ventilated thermocline}

Experiments with varying ridge depths (G4 and G5), but with the same channel width as G3, show how the meridional spreading is confined to the region below the height of the ridge (Fig. 14). Both of these produce quantitatively different patterns of deep stratification from $\mathrm{G} 3$, but the circulation pattern is qualitatively similar in all three cases. The $4^{\circ}$ isotherm outcrops approximately at the latitude separating the periodic channel from the closed domain and in the deep ocean forms the upper boundary to the deep stratification. In all these cases there is little stratification above this isotherm until one reaches the bottom of the main thermocline, as is most apparent from the lower panel of Fig. 14. Equatorward of the periodic channel, but still poleward of the division between the subtropical and subpolar gyre, surface water is entrained into the internal thermocline. Thus, whereas in a completely closed domain (G1) the temperature difference across the internal thermocline is equal to the surface temperature difference across the subpolar gyre, in the presence of a periodic channel the temperature difference across the thermocline is only the temperature difference between the subpolar/subtropical gyre boundary and the opening of the periodic channel. The abyssal stratification then takes up the remaining meridional temperature difference, with the stratification confined to a region below the top of the ridge.

This picture is consistent with the experiments with varying channel width (Fig. 15), where, to simplify matters, we consider only a single hemisphere. With a narrower channel (not shown) the results differ quantitatively but are consistent with the overall picture. The $4^{\circ}$ isotherm, for example, now outcrops equatorward of the periodic channel and is thus entrained into the main thermocline; the thermostad between the main thermocline and the abyssal thermocline is consequently cooler, the main thermocline is stronger, and the abyssal thermocline weaker. Perhaps surprisingly, the qualitative picture holds even as the channel is made very wide, but with interesting differences. Let us allow the channel to widen well beyond the latitude of zero wind stress curl. Now, in in a fully enclosed basin, of if the channel is poleward of the latitude of zero wind stress curl, the latitude of zero wind stress curl is the approximate source of mode water. There is an equatorward movement of fluid in the Ekman layer across the latitude of zero wind stress curl; this becomes the densest water entering the subtropical gyre, and it therefore must also be the density of the water at the top of the internal thermocline. Thus, a thick thermostad forms between the surface and this level. The temperature difference across the internal thermocline is then equal to the tem- perature difference across the subpolar gyre, and a ventilated thermocline calculation then determines the approximate structure of the thermocline along trajectories above the internal boundary layer.

If the channel extends equatorward past the latitude of zero wind stress curl, then that latitude has less special significance since water in the channel, in any case, sinks to the level of the sill before spreading equatorward. Rather, characteristics enter the ventilated thermocline from the surface only equatorward of the channel boundary, and deep thermostads form at this latitude, not the latitude of the zero wind stress curl. Compared to the case in which the channel boundary is poleward of the zero wind stress curl, the main (internal plus ventilated) thermocline is weakened, and the abyssal thermocline is strengthened, but otherwise the dynamics are similar to those described by ventilated thermocline theory. This is seen in Fig. 16, which plots the lower depth of the lowest layer of the ventilated thermocline in the two cases. In G3s this density is given by the water that outcrops at the line of zero wind stress curl. In the case with the wider channel this density is given by water that outcrops at the equatorward boundary of the channel. Both cases show characterisic features of the ventilated thermocline (e.g., Fig. 7 of Luyten et al. 1983); note, for example, the large region of almost uniform depth that stretches equatorward and westward from the eastern end of the line of subduction, although because of the presence of a region of Ekman upwelling at low latitudes in the wind forcing used here this cannot unambiguously be associated with a shadow zone, as in the classical theory.

An analytic calculation of a three-layer ventilated thermocline shows how robust the formation of a deep thermostad is, even as the latitude of subduction moves a long way equatorward. The calculation follows Luyten et al. (1983), with boundary conditions taken from the numerical simulation. In particular, the winds used are a low-order polynomial fit to those used to force the numerical simulation, and the outcrop latitudes and density differences across the layers are taken from the simulation itself. The abyssal density is taken to be that below the main thermocline, but above the abyssal thermocline. Then, specifically, layers corresponding to the densities of 1031.3, 1030.5, 1029, and 1028, with outcrop latitudes of $22^{\circ} \mathrm{S}$ and $31^{\circ} \mathrm{S}$ for the uppermost two layers, are used for the control case. Wider channels (going equatorward of the zero wind stress curl line) are emulated by quickly bringing the wind to zero poleward of the channel boundary, again by way of polynomial fits so that an analytic solution can be obtained. This serves to define the extreme latitude of trajectories entering the ventilated thermocline from the surface. The wind stress (and its curl) is altered only slightly equatorward of this. Layer densities of 1030.4, 1029.7, 1029, and 1028 for the wide channel are used - that is, only the densities of the deepest moving layer and of the abyss are altered, and the outcrop latitudes that are 
(a)

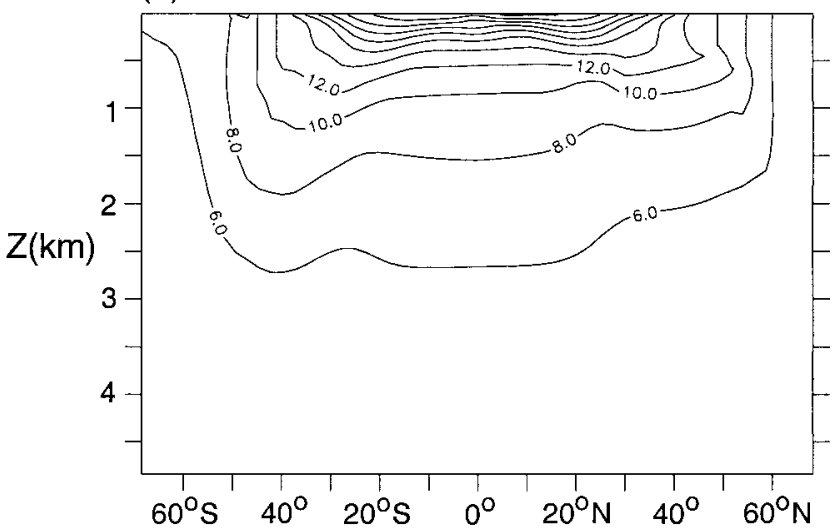

(c)

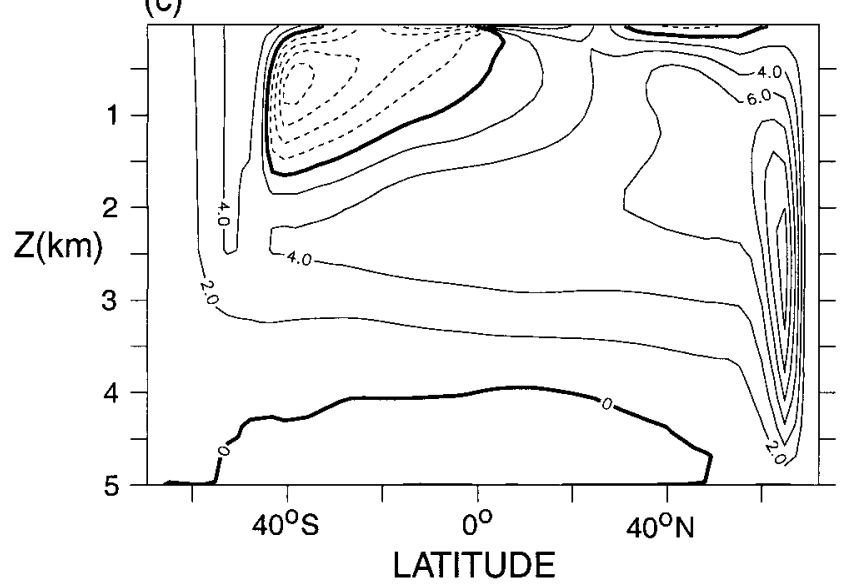

(b)

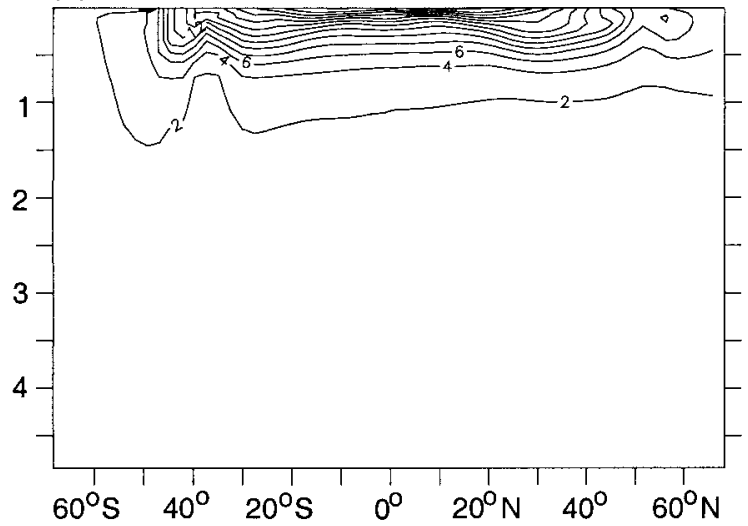

(d)

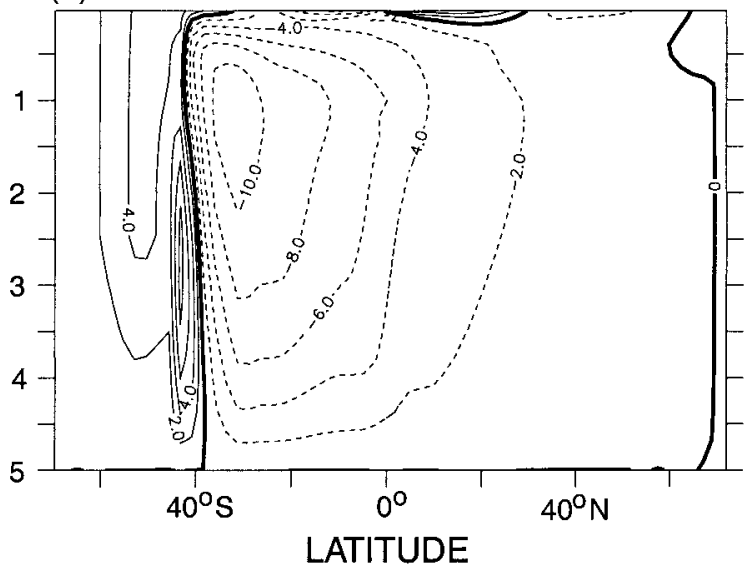

FIG. 21. Temperature sections (top) and meridional overturning circulations (bottom) from two integrations with the same geometry as G3 but neither of which have a temperature gradient across the periodic channel. On the left is G8 and on the right is G9.

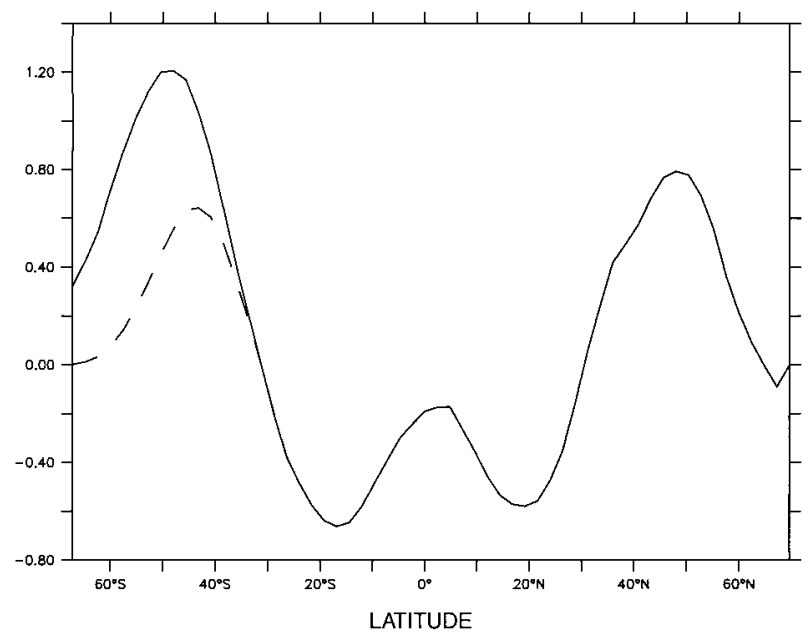

FIG. 22. The (zonally uniform) wind stress $\left(\mathrm{dyn} \mathrm{cm}^{-2}\right)$. The solid curve is zonally averaged Hellerman and Rosenstein (used in most experiments). The wind in the dashed curve differs only in the southern part of the domain, and is that used in expt G11. specified are left unaltered. The layer densities and the outcrop latitudes can be approximately specified a priori from a knowledge of the surface boundary conditions and the latitude of zero wind stress curl or channel edge, whichever is equatorward, although there appears to be no exact a priori criterion for the density of water below the main and above the abyssal thermocline. The analytic calculation also requires that the parameter $H_{0}$ be specified, this being the (constant) thickness of the deepest ventilated layer at the eastern boundary. Solutions of the analytic calculation are consistent with the full simulations (Fig. 17) in a number of aspects. Three cases are calculated, differing primarily in the latitude of the zero wind stress curl. The outcrop of thick thermostad (the deepest ventilated layer) and the southermost edge of the subtropical gyre move equatorward with the zero wind stress curl line, but the shallow region where the ventilated thermocline is most intense (i.e., where vertical gradients of density are strongest) is largely unaltered, except for a slight deepening present in both simulation and calculation. A deep thermostad is consistently reproduced in both numerical simulation and analytic calculation, even as its latitudinal extent is 
(a)

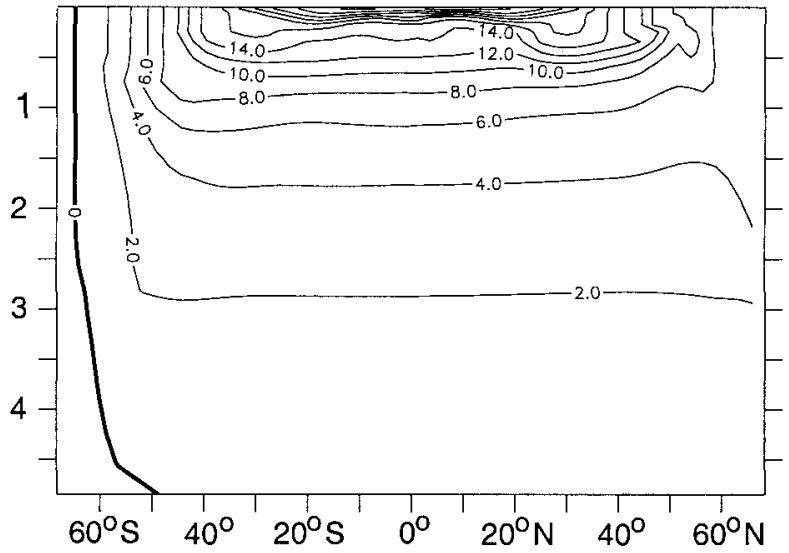

(c)

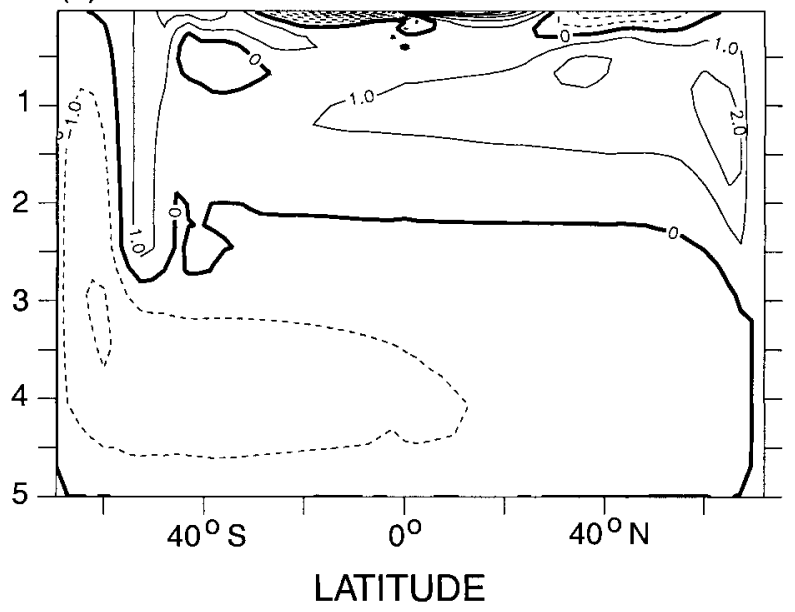

(b)

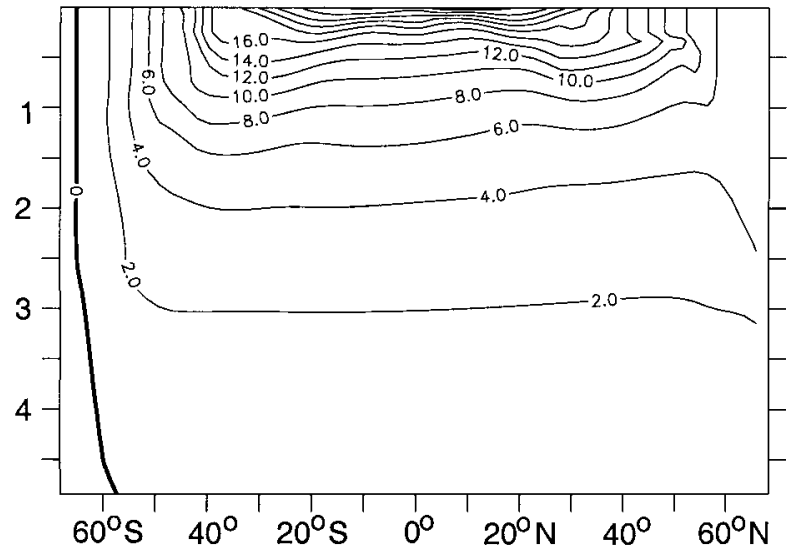

(d)

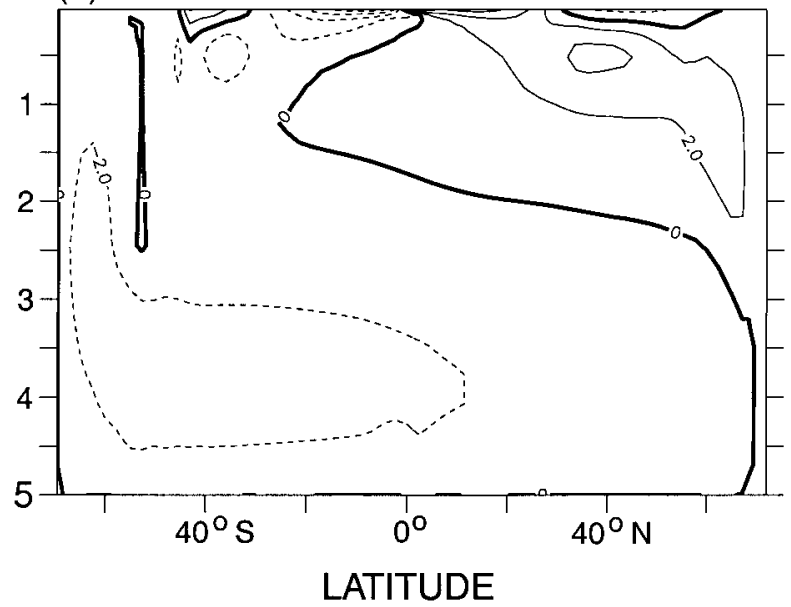

FIG. 23. Temperature sections (top) and meridional overturning circulation (bottom) from two integrations: Left column: G11-partially open Southern Hemisphere channel, but with reduced winds in the open channel. Right column: G12-i.e., same except that wind is zero over the Southern Hemisphere channel.

greatly reduced, and the pool of mode water is latitudinally compressed.

\section{d. Diffusivity scaling}

Does the diffusivity scaling for the thickness of the main internal thermocline still hold in the presence of an ACC and deep stratification? How does the thickness of the abyssal thermocline vary with diffusivity? Intuitively, one expects that at least the thickness of these thermoclines will monotonically fall with diffusivity since we still expect their dynamics to be governed by an advective-diffusive balance. However, the form of the scaling is not obvious. For example, suppose an advective-diffusive balance, like (3.5), with scales

$$
\frac{W \Delta T}{\delta} \sim k_{\nu} \frac{\Delta T}{\delta^{2}} .
$$

Then, if the meridional circulation (and thence $W$ ) were to be completely independent of $k_{\nu}$, for example, if it were entirely wind driven, the thermocline thickness would be expected to be proportional to the unit power of the diffusivity rather than the half power.

We examine this in the simplest dynamical setting of a single hemisphere with a partially blocked circumpolar channel. Profiles of the vertical derivative of temperature with varying values of diffusivity are illustrated in Fig. 19. The internal thermocline thickness continues to scale as the half-power of the diffusivity. The heat transport shows a similar scaling, but the results are less clean. (Some of these simulations, at higher values of diffusivity, show nearly periodic oscillations in the amplitude of the overturning circulation, with an amplitude of up to $10 \%$ of the peak, and the results shown reflect an average value. The thickness of the internal thermocline is, however, virtually constant in time.) Again, the thickness and the meridional heat transport at the smallest explicit value of the diffusivity $\left(0.1 \mathrm{~cm}^{2} \mathrm{~s}^{-1}\right)$ do not fall on the scaling lines: since the thermocline thickness asymptotes at small values of diffusivity, then 

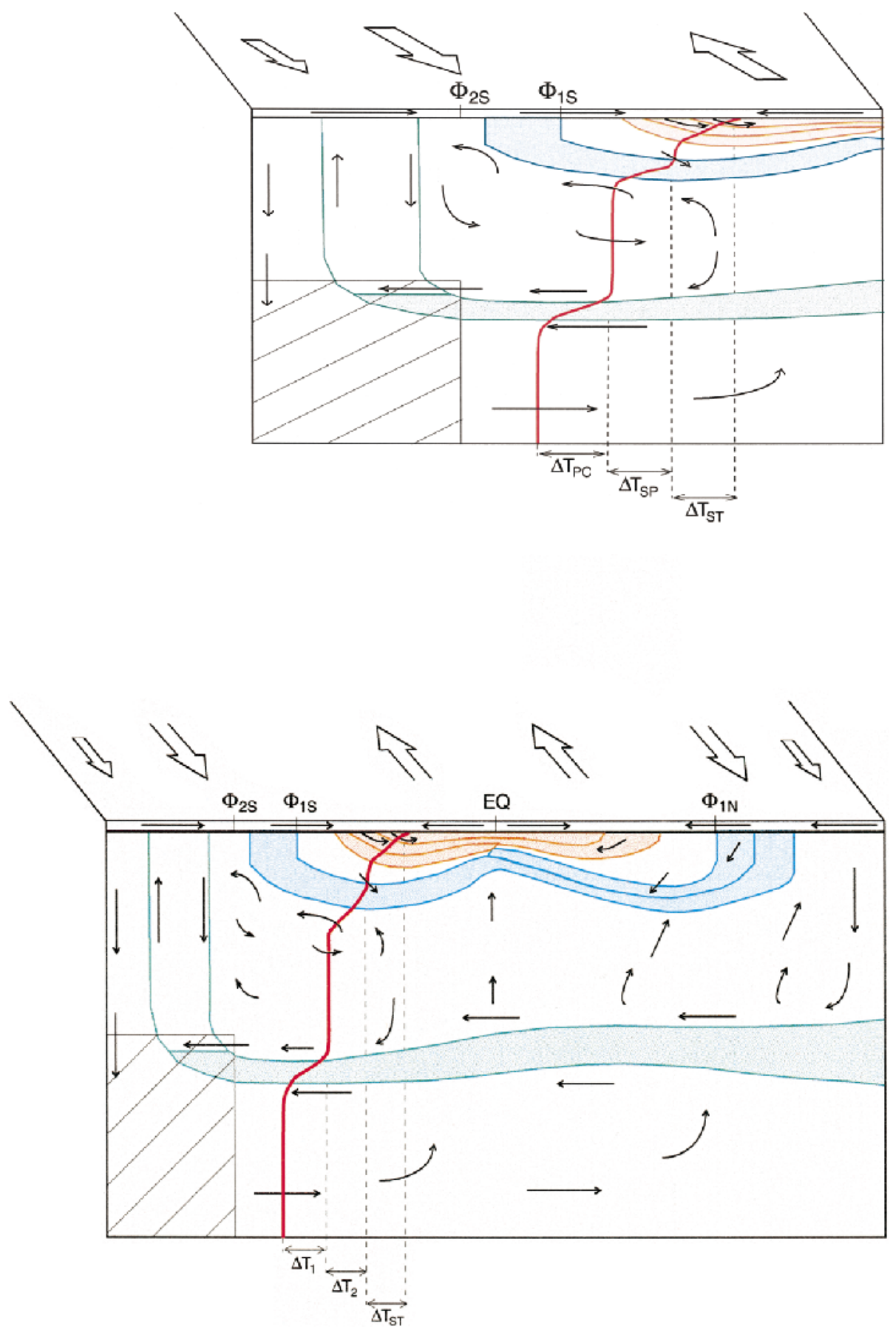

FIG. 24. Schema of the circulation in an idealized single-basin ocean with a periodic region, partially blocked by a thin, meridionally oriented topographic ridge in the Southern Hemisphere. Upper panel: circulation in a single hemisphere with a wall at the equator. Lower panel: two-hemisphere circulation. The hatched region in the lower left indicates the topographic ridge. $\Phi_{2 S}$ is the boundary of the periodic region, and $\Phi_{1 S}$ and $\Phi_{1 N}$ are the latitudes where the wind stress curl changes sign, i.e., the subtropicalsubpolar gyre boundaries. The wind forcing is indicated by the double arrows, and the temperature forcing is such that the surface buoyancy decreases poleward, with, in the two-hemisphere case, a colder Southern Hemisphere. The colored (shaded) regions indicate the different thermocline regimes-the amber (uppermost shaded) advective ventilated thermocline, the diffusive internal thermocline (blue, middle shaded) and diffusive abyssal thermocline (green, lower shaded). Mode water lies at the base of (and is part of) the ventilated thermocline and above the internal thermocline. The maximum of the abyssal thermocline lies near the level of the top of the topography. The thick (red) line is a temperature profile in the middle of the subtropical gyre, indicating how the surface meridional temperature gradient is mapped to the three thermoclines. In the single hemisphere case $\Delta T_{\mathrm{PC}}, \Delta T_{\mathrm{SP}}$, and $\Delta T_{\mathrm{ST}}$ are the 
(using 4.20) this probably reflects the presence of some small numerical diffusivity rather than a meridional transport that is actually becoming independent of diffusivity. However, still higher resolution experiments would be needed to definitively confirm this.

The structure, but not the amplitude, of the deep stratification is also a function of diffusivity. With a small value of diffusivity, various vertical profiles in the subtropical gyre (equatorward of the channel) are illustrated in Fig. 18. Note how dissimilar these are to those in Fig. 1; in particular, the vertical velocity changes sign at about the level of the abyssal thermocline, and there is an evident advective-diffusive balance at this level. Note too the similarity of the lower two profiles with the corresponding profiles in the similarity solutions in Fig. 7, except of course for the height of the internal boundary layer. (The advective-diffusive balance fails completely in the ventilated thermocline.) For small diffusivity and simple topographic blocking, the region of maximum abyssal stratification is concentrated at the height of the ridge and is clearly separated from the main thermocline. The thickness of this abyssal thermocline is well defined and diminishes as the diffusivity falls (Fig. 19). In the limit of zero diffusivity it will become a thin region of discontinuous temperature separating the two water masses, but the limit is singular and diffusivity term remains important. A scaling for the thickness of the abyssal thermocline can be constructed as in section $3 \mathrm{~b}$. The appropriate scaling is expected to be that of (3.6), (3.7), and (3.8), rather than (3.7), (38), and (311): the thermocline is not intrinsically sloping, so the appropriate horizontal scale is the external basin or gyre scale. That is, it is a constant, not a function of the solution itself, unlike the case with main internal thermocline. The correctness of the scaling is not self-evident, however, as the temperature gradient is not directly associated with thermal wind balance. If the reasoning is nevertheless correct, the abyssal thermocline thickness will scale as in (3.9) rather than (3.12), that is, as $\kappa^{1 / 3}$ and not $\kappa^{1 / 2}$. Figure 20 illustrates that this expectation is born out; if anything, the results show a dependence that is shallower than $\kappa^{1 / 3}$. (Of course the geometry does differ from that of the main thermocline, so the correct scaling is in fact by no means self-evident.) The strength of the meridional overturning circulation of the bottom cell also falls monotonically with diffusivity. In these simulations it does not appear to asymptote at some floor, as might have been expected if it were solely wind driven. However the strength of the cell does fall as the wind over the channel falls (Fig. 23), and it also falls with the meridional temperature gradient across the channel (Fig. 21), and is likely to be a multiplicative function of these factors.

\section{e. Effects of meridional temperature gradient}

According to the above discussion, the abyssal stratification (and the overturning circulation in abyss) relies directly on a meridional temperature gradient across the model ACC. This may be explicitly demonstrated by setting the atmospheric temperature gradient (i.e., the temperature to which the ocean relaxes) to zero. We do this in two ways; in G8 the temperature across the model channel is set to its value at its equatorward edge, and everywhere else is the same as in G3. In G9, the temperature difference between the equator and the equatorward edge of the channel is equal to the total temperature difference between the equator and the poleward edge of the channel in G3. Thus, the total meridional temperature difference is the same in the two experiments, but it is confined to the region equatorward of the channel in G9. The salinity forcing is left unaltered.

Neither G8 nor G9 are able to produce abyssal stratification as well as G3 (Fig. 21). In G9 the meridional temperature gradient is mapped entirely to the ventilated and internal thermocline, and the abyss completely homogenizes at the temperature of the coldest surface waters, which here come from the uniform-temperature periodic channel. The meridional overturning circulation is characterized by strong overturning at the channel edge and a single cell poleward of this. In G8 the coldest water is now produced in the Northern Hemisphere and the meridional overturning circulation reflects this, with intense motion at the northern end of the domain and most of the abyss being filled with Northern Hemisphere polar water.

\section{f. Effects of variable winds}

Given the dynamical picture painted above, it is possible to address the extent to which local winds over the model ACC are responsible or necessary for the production of deep stratification and the extent to which the deep meridional overturning circulation depends on these winds, questions raised by Toggweiler and Samuels (1995).

The meridional overturning circulation of bottom wa-

$\leftarrow$

across the periodic channel, the subpolar region, and the subtropical gyre, respectively. In the one-hemisphere case the thermostad between the main and the abyssal thermoclines (model "intermediate water") has the density of surface water near $\Phi_{2 S}$, the boundary of the periodic channel. In the two-hemisphere case high-latitude Northern Hemisphere water is denser than water at $\Phi_{25}$; hence the water immediately above the abyssal thermocline is Northern Hemisphere "Deep" Water and the temperature difference across the abyssal thermocline is correspondingly reduced. The single arrows show the meridional overturning circulation; the details of this in the two-hemisphere case, especially in the Southern Hemisphere, depend on the relative temperatures in the two hemispheres [cf. Fig. 1 of Welander (1971b) and Fig. 1 of Samelson and Vallis (1997b).] 
ter is dependent on the existence of winds over the channel; these winds are responsible for the production of the so-called Deacon cell. That is, the bottom water is ventilated in a generalized way in the channel in that for any nonzero overturning circulation the properties of the bottom water are largely determined by those at the channel surface. Thus, we might expect that existence of the abyssal thermocline will not depend on the presence of the wind over the channel, provided that bottom water is at least produced.

To test this conjecture, in experiment G11 the winds are reduced over the partially blocked Southern Hemisphere channel, but in all other respects the experiment is identical to G3. The wind is reduced smoothly by multiplying by a cosine function that has a value of unity just beyond the equatorward side of the channel, which reduces the value of the winds in the center of the channel by about a factor of 2. The control and reduced wind are illustrated in Fig. 22. In experiment G12, the winds are reduced abruptly to zero over the channel. Qualitatively, the results are similar to those of G3, and in particular deep stratification is certainly produced (Fig. 23). The meridional circulation again is dominated by two cells-a deep bottom-water-producing cell emanating from the Southern Hemisphere, overlaying which is a shallower cell of Northern Hemisphere water. Quantitatively, however, the strength of the deep meridional overturning circulation is weaker than with control winds (especially when the winds are zero), a result consistent with that of Toggweiler and Samuels (1995). The strength of this deep circulation cell thus presumably depends on both wind over the circumpolar channel and diffusivity, although the precise functional relationship of these factors remains obscure.

\section{Discussion and conclusions}

Solutions with a PE model support the notion that the "main" thermocline has two dynamical regimes: the upper regime is quasi adiabatic and quasi inviscid, and, insofar as the isotherms outcrop in regions of Ekman downwelling, corresponds to a ventilated thermocline. At the base of the ventilated thermocline is a region that is unavoidably diffusive; this is the internal thermocline. Between the internal and ventilated thermocline lies, in general, a thick thermostad where the stratification is small. Here, although our simulations are still somewhat idealized (in particular in the absence of a seasonal cycle) a correspondence of this thick model thermostad to observed $18^{\circ}$ Water can be made in the Northern Hemisphere.

Scaling arguments suggest that the thickness of the internal thermocline should be proportional to the onehalf power of the diffusivity, provided the Ekman pumping velocity dominates the internal velocity to the extent that the horizontal length scale of the internal thermocline can be differentiated from the externally imposed basin scale. The strength of the meridional overturning circulation should then also be proportional to the onehalf power of the diffusivity. However, if the wind stress is relatively weak or nonexistent (or the diffusivity high), theory suggests that the internal thermocline thickness and meridional overturning circulation should be proportional to the one-third and two-thirds power of the diffusivity, respectively. All these predictions are borne out by the numerical simulations and by solutions of a similarity equation. In particular, a sequence of simulations, varying the diffusivity but keeping wind fixed, are consistent with two different scaling regimes at lower and higher values of diffusivity.

The structure of the meridional overturning circulation and the deep stratification are indeed sensitive to the presence of periodic boundary conditions (a crude idealization of the Antarctic Circumpolar Current) at high latitudes. Deep stratification can be produced, even if the diffusivity is uniformly small, by introducing a partially blocked circumpolar channel. If the channel is partially blocked by topography, and if the water there is sufficiently dense, then the densest water will spread equatorward at the level of the top of the topography. Above the topography, where the channel is completely open, the geostrophic constraint prevents such efficient meridional spreading; thus, if there is no topography, the densest waters are trapped in the ACC. The circulation is illustrated schematically in Fig. 24. Surface water from near the equatorial end of the channel is largely entrained into the main internal thermocline, beneath which is a single thermostad extending to the top of the topographic ridge. The nature of the water between the abyssal thermocline and the main thermocline depends sensitively on the temperature difference between the densest water from the opposite hemisphere (model North Atlantic Deep Water) and the density of the water near the equatorial edge of the channel (model Antarctic Intermediate Water). All of the water in the periodic channel can extend meridionally as soon as it falls to the level of the ridge. The deep stratification thus maps the meridional temperature gradient across the channel and, in the limit of small diffusivity this region of stratification, is concentrated in a region of small vertical extent near the top of the ridge. That is, as the diffusivity tends to zero, the meridional temperature gradient is mapped discontinuously to a vertical temperature profile. In the absence of topography (i.e., given an completely open channel) no efficient polar spreading is possible because the zonally averaged meridional velocity is zero. Further, if the densest available water occurs in the opposite, completely enclosed, hemisphere then this water will completely fill the abyss, regardless of the presence or otherwise of a partially blocked channel.

In these simulations the source of stratification below the internal thermocline is not the downward diffusion of heat but, rather, the presence of different water masses in the abyss, a notion that is evocative of traditional descriptions of water masses (e.g., Schmitz 1995), as 
well as being consistent with more geographically complete (but highly diffusive) simulations such as Cox (1989) and England (1993). The results suggest that the abyssal ocean contains near-adiabatic homogeneous water masses in which diffusive effects are negligible, separated from each other by thin highly stratified diffusive layers analogous to the "internal" part of the main thermocline. The strength of the deep meridional overturning circulation depends on both diffusivity and the strength of the wind over the circumpolar channel. With a more complex geometry and topography, the structure of deep stratification may become correspondingly more complex and, if the abyssal mixing is large, these thin boundary layers will become diffusively thickened. Nevertheless, analogs of bottom, deep, and intermediate water can be produced with a very simple geometry, giving rise an intermittently stratified abyss with uniformly small values of diffusivity. There is no need for geographically distinct sources of water to produce such deep stratification-indeed, it can be produced in a single-basin, single-hemisphere integrationbut there must be a meridional temperature gradient across the topographically blocked periodic channel.

One final speculative note: At the last glacial maximum the deep overturning circulation is thought to have been weaker than today's, in spite of a likely larger equator-to-pole temperature gradient (Broecker 1983). The theory and simulations here suggest a possible resolution to this: the deep overturning circulation is particularly sensitive to the high-latitude meridional temperature gradient, not the overall meridional temperature gradient, because the deep circulation is buffered from the low-latitude gradient by the near-uniform temperature at the base of the subtropical main thermocline. At the last glacial maximum the surface temperature gradient at high latitudes was, in fact, weaker than it is today, in part because of a cover of sea ice.

Acknowledgments. I am grateful for many conversations and exchanges with Roger Samelson. I also thank Ron Pacanowski and Steve Griffies for answering all my MOM questions and for their input, Kirk Bryan for discussion, and the reviewers for their critical comments. This work is funded by the NSF.

\section{REFERENCES}

Broecker, W., 1983: The Glacial World According to Wally. Columbia University, $482 \mathrm{pp}$.

Bryan, F., 1987: Parameter sensitivity of primitive equation ocean general circulation models. J. Phys. Oceanogr., 17, 970-985

Colin de Verdiere, A., 1986: On mean flow instabilities within the planetary geostrophic equations. J. Phys. Oceanogr., 16, 19811984.

_ 1988: Buoyancy driven planetary flows. J. Mar. Res., 46, 215265.

- 1989: On the interaction of wind and buoyancy driven gyres. J. Mar. Res., 47, 595-633.

Cox, M., 1989: An idealized model of the world ocean. Part I: The global-scale water masses. J. Phys. Oceanogr., 19, 1730-1752.
, and K. Bryan, 1984: A numerical model of the ventilated thermocline. J. Phys. Oceanogr., 14, 674-687.

Cummins, P., 1991: The deep water stratification of ocean general circulation models. Atmos.-Ocean, 19, 563-575.

— A. Gargett, and G. Holloway, 1990: Sensitivity of the GFDL ocean general circulation model to a parameterization of vertical diffusivity. J. Phys. Oceanogr., 20, 817-830.

England, M. H., 1993: Representing the global-scale water masses in ocean general circulation models. J. Phys. Oceanogr., 23, $1523-1552$.

Gill, A., and K. Bryan, 1971: Effects of geometry on the circulation of a three-dimensional southern hemisphere ocean model. DeepSea Res., 18, 685-721.

Gregg, M., 1987: Diapycnal mixing in the thermocline: A review. $J$. Geophys. Res., 92, 5249-5286.

Hellerman, S., and M. Rosenstein, 1983: Normal monthly wind stress over the world ocean with error estimates. J. Phys. Oceanogr., 13, 1093-1104.

Huang, R. X., 1988: On boundary value problems of the ideal-fluid thermocline. J. Phys. Oceanogr., 18, 619-641.

Ledwell, J., A. Watson, and C. Law, 1993: Evidence for slow mixing across the pycnocline from an open-ocean tracer-release experiment. Nature, 364, 701-703.

Luyten, J., J. Pedlosky, and H. Stommel, 1983: The ventilated thermocline. J. Phys. Oceanogr., 13, 292-309.

Marotzke, J., 1997: Boundary mixing and the dynamics of threedimensional thermohaline circulations. J. Phys. Oceanogr., 27, $1713-1728$.

McCartney, M., 1982: The subtropical recirculation of Mode Waters. J. Mar. Res., 40 (Suppl.), 427-464.

, and L. Talley, 1982: The subpolar mode water of the North Atlantic Ocean. J. Phys. Oceanogr., 12, 1169-1188.

Pacanowski, R., Ed., 1996: MOM 2: Documentation, user's guide and reference manual. GFDL Ocean Tech. Rep. 3.2, Geophysical Fluid Dynamics Laboratory, 329 pp. [Available from GFDL, Princeton, NJ 08542.]

Park, Y., and K. Bryan, 2000: Comparison of thermally driven circulations from a depth coordinate model and an isopycnal model. Part I: A scaling law and sensitivity to vertical diffusivty. $J$. Phys. Oceanogr., 30, 590-605.

Polzin, K. L., J. M. Toole, J. R. Ledwell, and R. W. Schmitt, 1997: Spatial variability of turbulent mixing in the abyssal ocean. Science, 276, 93-96.

Salmon, R., 1986: A simplified linear ocean circulation theory. $J$. Mar. Res., 44, 695-711.

_ 1990: The thermocline as an "internal boundary layer." J. Mar. Res., 48, 437-469.

Samelson, R. M., 1998: Large-scale circulation with locally enhanced vertical mixing. J. Phys. Oceanogr., 28, 712-726.

_ 1999: Geostrophic circulation in a rectangular basin with a circumpolar connection. J. Phys. Oceanogr., 29, 3175-3184.

_ _ and G. K. Vallis, 1997a: A simple friction and diffusion scheme for planetary geostrophic basin models. J. Phys. Oceanogr., 27, 186-194.

— diffusion: The two-thermocline limit. J. Mar. Res., 55, 223-275.

Schmitz, W. J., Jr., 1995: On the interbasin-scale thermohaline circulation. Rev. Geophys., 33, 151-173.

Smith, S., and G. K. Vallis, 1999: Linear baroclinic instability in extended regime geostrophic models. J. Atmos. Sci., 56, 15791593.

Stommel, H., and A. Arons, 1960: On the abyssal circulation of the world ocean-I. Stationary planetary flow patterns on a sphere. Deep-Sea Res., 6, 140-154.

— tions in a subtropical gyre. J. Mar. Res., 44, 695-711.

Toggweiler, J. R., and B. Samuels, 1995: Effect of Drake Passage on the global thermohaline circulation. Deep-Sea Res., 42, 477500 . 
Toole, J., K. Polzin, and R. Schmitt, 1994: Estimates of diapycnal mixing in the abyssal ocean. Science, 264, 1120-1123.

Veronis, G., 1969: On theoretical models of the thermocline circulation. Deep-Sea Res., 16 (Suppl.), 301-323.

- 1973: Model of the world ocean circulation: I, wind-driven, two-layer. J. Mar. Res., 31, 228-288.

Welander, P., 1959: An advective model of the ocean thermocline. Tellus, 11, 309-318.

, 1971a: Some exact solutions to the equations describing an ideal-fluid thermocline. J. Mar. Res., 21, 60-68.
— 1971b: The thermocline problem. Philos. Trans. Roy. Soc. London, 270A, 415-421.

Winton, M., 1996: The role of horizontal boundaries in parameter sensitivity and decadal-scale variability of coarse-resolution ocean general circulation models. J. Phys. Oceanogr., 26, 289-304.

Young, W. R., and G. Ierley, 1986: Eastern boundary conditions and weak solutions of the ideal thermocline equations. J. Phys. Oceanogr., 16, 1884-1900.

Zhang, S., C. A. Lin, and R. J. Greatbatch, 1992: A thermocline model for ocean-climate studies. J. Mar. Res., 50, 99-124. 